\title{
Knowledge-Aided STAP Using Low Rank and Geometry Properties
}

\author{
Zhaocheng Yang, ${ }^{1}$ Rodrigo C. de Lamare, ${ }^{2}$ Xiang Li, ${ }^{1}$ and Hongqiang Wang ${ }^{1}$ \\ ${ }^{1}$ Research Institute of Space Electronics, Electronics Science and Engineering School, National University of Defense Technology, \\ Changsha 410073, China \\ ${ }^{2}$ Communications Research Group, Department of Electronics, University of York, York YO10 5DD, UK
}

Correspondence should be addressed to Zhaocheng Yang; yangzhaocheng@gmail.com

Received 27 April 2014; Accepted 17 July 2014; Published 12 August 2014

Academic Editor: Hang $\mathrm{Hu}$

Copyright (C) 2014 Zhaocheng Yang et al. This is an open access article distributed under the Creative Commons Attribution License, which permits unrestricted use, distribution, and reproduction in any medium, provided the original work is properly cited.

This paper presents knowledge-aided space-time adaptive processing (KA-STAP) algorithms that exploit the low-rank dominant clutter and the array geometry properties (LRGP) for airborne radar applications. The core idea is to exploit the clutter subspace that is only determined by the space-time steering vectors, by employing the Gram-Schmidt orthogonalization approach to compute the clutter subspace. Simulation results illustrate the effectiveness of our proposed algorithms.

\section{Introduction}

Space-time adaptive processing (STAP) is considered an efficient tool for detection of slow targets by airborne radar systems in strong clutter environments [1-4]. However, due to the very high degrees of freedom (DoFs), the full-rank STAP has a slow convergence and requires about twice the DoFs of the independent and identically distributed (IID) training snapshots to yield an average performance loss of roughly $3 \mathrm{~dB}[1]$. In real scenarios, it is hard to obtain so many IID training snapshots, especially in heterogeneous environments. Reduced-dimension/reduced-rank methods [1-10], parametric adaptive matched filter (PAMF) [11], and sparse beamformers $[12,13]$ have been considered to counteract the slow convergence of the full-rank STAP. However, it still fundamental for radar systems to improve the convergence performance of STAP algorithms or reduce their required sample support in heterogeneous environments because the number of required snapshots is large relative to those needed in IID scenarios. By exploiting the sparsity of the received signal, sparsity-based STAP algorithms are developed to reduce the sample support [14-16]. However, these algorithms usually require high computation complexity.
Recently developed knowledge-aided (KA) STAP algorithms have received a growing interest and become a key concept for the next generation of adaptive radar systems (see [17-30] and the reference therein). The core idea of KA-STAP is to incorporate prior knowledge, provided by digital elevation maps, land cover databases, road maps, global positioning system (GPS), previous scanning data, and other known features, to compute estimates of the clutter covariance matrix (CCM) with high accuracy $[17,18,24]$. Among the developed KA-STAP algorithms, they can be categorized into two cases: intelligent training and filter selection; and Bayesian filtering and data prewhitening [17]. In the former case, prior knowledge of the interference environments is used for the filter selection or the selection of the so-called filter training data. By using database information, [25] identifies and screens roadways from the training set and provides approximately $15 \mathrm{~dB}$ gains compared with no prior knowledge. Digital land classification data and digital elevation data are used to characterize clutter patches for secondary data selection and ensure independent secondary data samples [26]. Additionally, a geographic-map-aided radar detector is exploited to remove dynamic outliers from the training data [27]. In the latter case, prior knowledge 
is incorporated directly by the filter to aid in suppressing the clutter. Colored loading (CL) implements the STAP filter in two steps: a prewhitening step using the prior matrix, followed by adaptive filtering [28]. Fully automatic methods for combining the matrix with prior knowledge and secondary data are considered in [23, 29]. Furthermore, in [30], an automatic combination of the inverse of the matrix with prior knowledge and the inverse of the estimated covariance matrix from secondary data is developed. Moreover, there is a class of approaches that exploit prior knowledge of the clutter ridge to form the STAP filter weights in [19-22]. The authors in [19] introduced a knowledge-aided parametric covariance estimation (KAPE) scheme by combining both prior knowledge and data observations within a parameterized model to capture instantaneous characteristics of the cell under test (CUT). A modified sample matrix inversion (SMI) procedure to estimate the CCM using a least-squares (LS) approach has been described in [20] to overcome the range-dependent clutter nonstationarity in conformal array configurations. However, both approaches require the pseudoinverse calculation to estimate the CCM and this often requires a computationally costly singular value decomposition (SVD) [19]. Although two weighting approaches with lower computations are discussed in [19], they are suboptimal approaches to the LS estimator (LSE) computed by the SVD and the performance of these approaches depends on the radar system parameters, especially the array characteristics [19]. Moreover, the latter approach has not considered the situation when prior knowledge has uncertainties. Under the assumption of the known clutter ridge in the angle-Doppler plane, the authors in [21] imposed a sparse regularization to estimate the clutter covariance excluding the clutter ridge. Although this kind of method can obtain a high resolution even with only one snapshot, it requires the designer to know the exact positions of the clutter ridge resulting in being sensitive to prior knowledge. Furthermore, the computational complexity caused by sparse recovery is expensive. A data independent STAP method based on prolate spheroidal wave functions (PSWF) has been considered in MIMO radar by incorporating the clutter ridge [22], where the computational complexity is significantly reduced compared with the approaches in $[19,20]$. However, it is highly dependent on the ideal clutter subspace and is not robust against clutter subspace mismatches.

In this paper, we propose KA-STAP algorithms using prior knowledge of the clutter ridge that avoid the pseudoinverse calculation, require a low computational complexity, and mitigate the impact of uncertainties of prior knowledge. Specifically, for a side-looking uniformly spaced linear array (ULA), the proposed method selects a group of linearly independent space-time steering vectors that can represent the ideal clutter subspace using prior knowledge of the dominant low-rank clutter and the array geometry properties (LRGP). The orthogonal bases of the clutter ideal subspace are computed by a Gram-Schmidt orthogonalization procedure on the selected space-time steering vectors. Two robust approaches to compute the STAP filter weights are then presented based on the estimated clutter subspace. To overcome the performance degradation caused by the internal clutter motion (ICM), we employ a covariance matrix taper (CMT) to the estimated CCM. The array calibration methods discussed in [19] can be applied to our proposed algorithm to mitigate the impact of nonideal factors, such as channel mismatch. Moreover, a reduced-dimension version of the proposed KA-STAP algorithm is devised for practical applications. Finally, simulation results demonstrate the effectiveness of our proposed algorithms.

The work is organized as follows. Section 2 introduces the signal model in airborne radar applications. Section 3 details the approach of the proposed KA-STAP algorithms and also discusses the computational complexity. The simulated airborne radar data are used to evaluate the performance of the proposed algorithms in Section 4. Section 5 provides the summary and conclusions.

\section{Signal Model}

The system under consideration is a side-looking pulsed Doppler radar with a ULA consisting of $M$ elements on the airborne radar platform, as shown in Figure 1. The platform is at altitude $h_{p}$ and moving with constant velocity $v_{p}$. The radar transmits a coherent burst of pulses at a constant pulse repetition frequency $(\mathrm{PRF}) f_{r}=1 / T_{r}$, where $T_{r}$ is the pulse repetition interval (PRI). The transmitter carrier frequency is $f_{c}=c / \lambda_{c}$, where $c$ is the propagation velocity and $\lambda_{c}$ is the wavelength. The number of pulses in a coherent processing interval (CPI) is $N$. The received signal from the iso-range of interest is represented by a space-time $N M \times 1$ data vector $\mathbf{x}$.

The received space-time clutter plus noise return from a single range bin can be represented by [4]

$$
\mathbf{x}=\sum_{m=1}^{N_{a}} \sum_{n=1}^{N_{c}} \sigma_{m, n} \mathbf{v}\left(f_{s, m, n}, f_{d, m, n}\right) \odot \boldsymbol{\alpha}(m, n)+\mathbf{n},
$$

where $\mathbf{n}$ is the Gaussian white thermal noise vector with the noise power $\sigma_{n}^{2}$ on each channel and pulse, $N_{a}$ is the number of range ambiguities, $N_{c}$ is the number of independent clutter patches over the iso-range of interest, $f_{s, m, n}$ and $f_{d, m, n}$ are the spatial and Doppler frequencies of the $m n$th clutter patch, respectively, $\sigma_{m, n}$ is the complex amplitude for the $m n$th clutter patch, $\boldsymbol{\alpha}(m, n)=\boldsymbol{\alpha}_{d}(m, n) \otimes \boldsymbol{\alpha}_{s}(m, n)$ is the space-time random taper vector characterizing the voltage fluctuation caused by nonideal factors, such as ICM and channel mismatch (where $\boldsymbol{\alpha}_{d}(m, n)$ and $\boldsymbol{\alpha}_{s}(m, n)$ are the temporal and spatial random tapers), and $\mathbf{v}\left(f_{s, m, n}, f_{d, m, n}\right)$ is the $N M \times 1$ space-time steering vector for a clutter patch with $f_{s, m, n}$ and $f_{d, m, n}$.

The space-time steering vector is given as the Kronecker product of the temporal and spatial steering vectors, $\mathbf{v}\left(f_{s, m, n}, f_{d, m, n}\right)=\mathbf{v}_{t}\left(f_{d, m, n}\right) \otimes \mathbf{v}_{s}\left(f_{s, m, n}\right)$, which are given by [1]

$$
\begin{aligned}
& \mathbf{v}_{t}\left(f_{d, m, n}\right)=\left[1, \ldots, \exp \left(j 2 \pi(N-1) f_{d, m, n}\right)\right]^{T}, \\
& \mathbf{v}_{s}\left(f_{s, m, n}\right)=\left[1, \ldots, \exp \left(j 2 \pi(M-1) f_{s, m, n}\right)\right]^{T},
\end{aligned}
$$

where ()$^{T}$ denotes the transpose operation, $f_{s, m, n}=$ $d_{a} / \lambda_{c} \cos \theta_{m, n} \sin \phi_{m, n}, f_{d, m, n}=2 v_{p} T_{r} / \lambda_{c} \cos \theta_{m, n} \sin \phi_{m, n}$, 


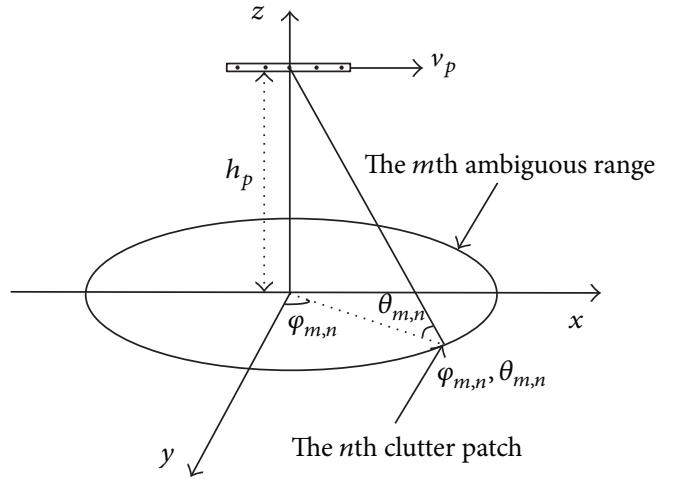

FIGURE 1: Airborne radar geometry with a ULA antenna.

and $d_{a}$ is the intersensor spacing of the ULA. If we stack all clutter patches' amplitudes into a vector

$$
\boldsymbol{\sigma}=\left[\sigma_{1,1}, \ldots, \sigma_{1, N_{c}}, \ldots, \sigma_{N_{a}, 1}, \ldots, \sigma_{N_{a}, N_{c}}\right]^{T}
$$

and assume there are no nonideal factors, then the clutter plus noise received data denoted by (1) can be described as

$$
\mathbf{x}=\mathbf{x}_{c}+\mathbf{n}=\mathbf{V} \boldsymbol{\sigma}+\mathbf{n},
$$

where $\mathbf{V}$ denotes the clutter space-time steering matrix, given by

$$
\begin{aligned}
& \mathbf{V}=\left[\mathbf{v}\left(\phi_{1,1}, \theta_{1,1}, f_{1,1}\right), \ldots,\right. \\
& \mathbf{v}\left(\phi_{1, N_{c}}, \theta_{1, N_{c}}, f_{1, N_{c}}\right), \ldots, \\
& \mathbf{v}\left(\phi_{N_{a}, 1}, \theta_{N_{a}, 1}, f_{N_{a}, 1}\right), \ldots, \\
& \left.\mathbf{v}\left(\phi_{N_{a}, N_{c}}, \theta_{N_{a}, N_{c}}, f_{N_{a}, N_{c}}\right)\right] .
\end{aligned}
$$

Thus, the CCM based on (4) can be expressed as

$$
\mathbf{R}_{c}=\mathbf{V} \boldsymbol{\Sigma} \mathbf{V}^{H}
$$

where $\boldsymbol{\Sigma}=E\left[\boldsymbol{\sigma} \boldsymbol{\sigma}^{H}\right]$. Under the condition that the clutter patches are independent from each other, $\Sigma=\operatorname{diag}(\mathbf{a})$, $\mathbf{a}=\left[a_{1,1}, a_{1,2}, \ldots, a_{N_{a}, N_{c}}\right]^{T}$, and $a_{m, n}=E\left[\left|\sigma_{m, n}\right|^{2}\right](m=$ $1, \ldots, N_{a}, n=1, \ldots, N_{c}$ ) for the statistics of the clutter patches. Here, $E[\cdot]$ denotes the expectation operator, $\operatorname{diag}(\mathbf{a})$ stands for a diagonal matrix with the main diagonal taken from the elements of the vector a, and ()$^{H}$ represents the conjugate transpose of a matrix.

The optimal filter that maximizes the output SINR for the Gaussian distribution clutter which is given by the full-rank STAP processor can be written as [4]

$$
\mathbf{w}_{\mathrm{opt}}=\mu \mathbf{R}^{-1} \mathbf{s},
$$

where $\mu$ is a constant which does not affect the SINR performance, $\mathbf{s}$ is the $N M \times 1$ space-time steering vector in the target direction, and $\mathbf{R}=E\left[\mathbf{x} \mathbf{x}^{H}\right]=\mathbf{R}_{c}+\sigma_{n}^{2} \mathbf{I}$ is the clutter plus noise covariance matrix ( $\mathrm{I}$ is the identity matrix).

\section{KA-STAP Algorithms Using LRGP}

In this section, we firstly review the method that estimates the CCM using an LS technique in $[19,20]$ and then detail the design and the computational complexity of the proposed KA-STAP algorithms using LRGP. The key difference between the proposed and existing algorithms lies in the way to compute the CCM.

3.1. CCM Estimated by LS. In practice, prior knowledge of certain characteristics of the radar system and the aerospace platform, such as platform heading, speed and altitude, array normal direction, and antenna phase steering, can be obtained from the Inertial Navigation Unit (INU) and the GPS data [19]. In other words, we can obtain the values of the number of range ambiguities $N_{a}$, the platform velocity $v_{p}$, and the elevation angle $\theta$. Thus, we can develop KA-STAP algorithms based on this prior knowledge, for example, the methods described in [19-22]. In reality, the clutter consists of returns over a continuous geographical region, which we divide into a discrete set of clutter patches for analytical and computational convenience. The rest of the discussion is on the issues associated with choosing the number of clutter patches $N_{c}$. A possible approach is to assume a value of $N_{c}$ and discretize the whole azimuth angle evenly into $N_{c}$ patches for each range bin $[19,20]$. In addition, it usually ignores range ambiguities, that is, $N_{a}=1$, where the justification can be seen in [19]. Then, the parameter $\sigma$ in (4) can be estimated using the observation data by solving the LS problem as follows $[19,20]$ :

$$
\widehat{\boldsymbol{\sigma}}=\arg \min _{\boldsymbol{\sigma}}\|\mathbf{x}-\mathbf{V} \boldsymbol{\sigma}\|^{2}
$$

where $\hat{\boldsymbol{\sigma}}=\left[\widehat{\sigma}_{1}, \widehat{\sigma}_{2}, \ldots, \widehat{\sigma}_{N_{c}}\right]^{T}$. Herein, the solution for the above problem based on an LS technique is given by

$$
\widehat{\boldsymbol{\sigma}}=\left[\mathbf{V}^{H} \mathbf{V}\right]^{-1} \mathbf{V}^{H} \mathbf{x} .
$$

To avoid the effect of the target signal at CUT, the near range bins of the CUT are used to estimate $\sigma$ [20], which is given by

$$
\widehat{\sigma}_{m, n}^{2}=\frac{1}{L} \sum_{l=1}^{L}\left|\widehat{\sigma}_{m, n ; l}\right|^{2},
$$

where $L$ is the total number of the secondary data. Then, the estimated CCM by the LS method (we call it LSE in the following) is

$$
\widehat{\mathbf{R}}_{c}=\mathbf{V} \widehat{\mathbf{\Sigma}} \mathbf{V}^{H}
$$

Then the clutter plus noise covariance matrix is estimated as

$$
\widehat{\mathbf{R}}=\widehat{\mathbf{R}}_{c}+\widehat{\sigma}_{n}^{2} \mathbf{I},
$$

where $\widehat{\sigma}_{n}^{2}$ is the estimated noise power level which can be collected by the radar receiver when the radar transmitter operates in a passive mode [2]. Finally, the STAP filter weights can be computed according to (7) using $\widehat{\mathbf{R}}$ instead of $\mathbf{R}$. 
It should be noted that it often assumes a large number of clutter patches $N_{c}$ to obtain a good estimation accuracy of the estimated CCM. Specifically, if the assumed number of clutter patches $N_{c}>N M$, then $\left[\mathbf{V}^{H} \mathbf{V}\right]^{-1}$ does not exist and has to be calculated by the pseudoinversion. However, the computational complexity of the terms $\left[\mathbf{V}^{H} \mathbf{V}\right]^{-1}$ is very high; that is, $O\left(\left(N_{c}\right)^{3}\right)+O\left(N_{c}(N M)^{2}\right)$, which should be avoided in practice. Two suboptimal approximation weighting approaches with lower cost are discussed in [19]. But the performance of these approaches depends on the radar system parameters [19]. In addition, in the presence of nonideal factors in the clutter component and despite the inclusion of the estimated angle-independent channel mismatch in the space-time steering vectors $\mathbf{V}$ and the use of the modified V to solve the problem (8), the techniques do not consider the impact of the temporal random taper $\boldsymbol{\alpha}_{d}$. Nevertheless, the received data vector $\mathbf{x}$ is formed by all nonideal factors. Thus, whether it is suitable to compute the parameter $\boldsymbol{\sigma}$ only considering the spatial random taper is worth being investigated, as will be discussed in Section 3.3.

3.2. Proposed KA-STAP Algorithm. To overcome the rank deficiency and the inverse of the matrix $\mathbf{V}^{H} \mathbf{V}$, in the following, we will detail the proposed KA-STAP algorithm to estimate the CCM using prior knowledge of LRGP. In this subsection, we only consider the ideal case of the received data, that is, the signal model in (4).

From (4), we know that the clutter return is a linear combination of returns from all clutter patches. Thus, we have

$$
\operatorname{span}\left(\mathbf{R}_{c}\right)=\operatorname{span}(\mathbf{V})=\operatorname{span}\left(\mathbf{V} \mathbf{V}^{H}\right) .
$$

From (13), it can be seen that the clutter subspace is independent of the power of the clutter patches and is only determined by the clutter space-time steering vectors. Thus, the orthogonal basis of the clutter subspace $\mathbf{U}$ can be calculated by $\mathbf{V}$ or $\mathbf{V} \mathbf{V}^{H}$. The other problem to calculate the clutter subspace arising is that one should know the clutter rank first. Fortunately, some rules for estimating the clutter rank were discussed in previous literature, such as $[1,2,31,32]$. Specifically, for a side-looking ULA, the estimated clutter rank is approximated by Brennan's rule as

$$
\operatorname{rank}\left(\mathbf{R}_{c}\right) \approx N_{r}=\lceil M+\beta(N-1)\rceil,
$$

where $\beta=2 v_{p} T_{r} / d_{a}$ and the brackets \lceil\rceil indicate rounding to the nearest largest integer. In [32], this rule has been extended to the case with arbitrary arrays. Usually, $N_{r} \ll$ $N M$ and the STAP algorithms can be performed in a low dimensional space so that the computational complexity and the convergence can be significantly improved [22].

After the clutter rank is determined, a low-complexity approach to computing the orthogonal bases of the clutter subspace is to perform the Gram-Schmidt orthogonalization procedure on the space-time steering vectors $\mathbf{V}$, where the implementation steps of the Gram-Schmidt orthogonalization are listed in Algorithm 1 and interested readers are referred to [33] for further details. Note that this procedure is at the computational cost of $O\left(\left(N_{c}+1\right) N_{c} N M / 2\right) \ll O\left(N_{c}^{3}+\right.$

\section{Initialization: \\ $\beta=2 v_{p} T_{r} / d_{a}, N, M, \widehat{\sigma}_{n}^{2}$.}

Select a group of space-time steering vectors

(for side-looking ULA)

$$
\begin{aligned}
& \left\{\overline{\mathbf{v}}_{p}\right\}_{p=0}^{N_{r}}, \\
& \text { where } \overline{\mathbf{v}}_{p}(n, m)=\exp \left(j 2 \pi f_{s}(\beta n+m)\right), \\
& N_{r}=M+\beta(N-1) \text {, and } f_{s}=\frac{p}{N_{r}}, p=0,1, \ldots, N_{r}-1,
\end{aligned}
$$

\section{Compute calibrated space-time steering vectors}

Estimate $\widehat{\boldsymbol{\alpha}}_{s}$ using the methods in [19],

where columns of $\widehat{\boldsymbol{\Xi}}_{s}$ are all equivalent to $\mathbf{1}_{N} \otimes \widehat{\boldsymbol{\alpha}}_{s}$, $\mathbf{V}_{s}=\mathbf{V} \odot \widehat{\Xi}_{s}$,

(In RD version, $\overline{\mathbf{V}}_{s}=\mathbf{S}_{D}^{H} \mathbf{V}_{s}$ ),

$$
\begin{aligned}
& \text { Compute } \mathbf{U}_{s} \\
& \begin{array}{l}
\mathbf{u}_{s, 0}=\overline{\mathbf{v}}_{s, 0} /\left\|\overline{\mathbf{v}}_{s, 0}\right\|, \\
\widetilde{\mathbf{u}}_{s, p}=\overline{\mathbf{v}}_{s, p}-\sum_{i=0}^{p-1}\left(\mathbf{u}_{s, i}^{H} \overline{\mathbf{v}}_{s, p}\right) \mathbf{u}_{s, i}, \\
\mathbf{u}_{s, p}=\widetilde{\mathbf{u}}_{s, p} /\left\|\tilde{\mathbf{u}}_{s, p}\right\|, p=1, \ldots, N_{r}-1, \\
\mathbf{U}_{s}=\left[\mathbf{u}_{s, 0}, \ldots, \mathbf{u}_{s, N_{r}-1}\right] . \\
\left(\text { In RD version, } \overline{\mathbf{U}}_{s} \text { instead of } \mathbf{U}_{s}\right),
\end{array}
\end{aligned}
$$

For each snapshot $l=1, \ldots, L$

$$
\widehat{\gamma}_{l, s}=\mathbf{U}_{s}^{H} \mathbf{x}_{l},
$$

Compute $\widehat{\mathbf{R}}_{\mathrm{c}}$

$$
\begin{aligned}
& \widehat{\boldsymbol{\Gamma}}=\operatorname{diag}\left(\frac{1}{L} \sum_{l=1}^{L} \widehat{\gamma}_{l, s} \odot \widehat{\gamma}_{l, s}^{*}\right), \\
& \widehat{\mathbf{R}}_{s}=\widehat{\mathbf{U}}_{s} \widehat{\boldsymbol{\Gamma}}_{s} \widehat{\mathbf{U}}_{s}^{H}, \\
& \text { Estimate } \widehat{\mathbf{T}}_{d}, \\
& \widehat{\mathbf{R}}_{c}=\widehat{\mathbf{R}}_{s} \odot \widehat{\mathbf{T}}_{d},
\end{aligned}
$$

$$
\text { (In RD version, } \overline{\mathbf{U}}_{s} \text { instead of } \mathbf{U}_{s}, \widehat{\overline{\mathbf{T}}}_{d} \text { instead of } \widehat{\mathbf{T}}_{d} \text { ) }
$$

\section{Compute U}

Adopt the Lanczos algorithm to $\widehat{\mathbf{R}}_{c}$ to compute $\mathbf{U}$, (In RD version, $\widehat{\overline{\mathbf{R}}}_{c}$ instead of $\widehat{\mathbf{R}}_{c}$ ),

Filter weights computation

$$
\widehat{\mathbf{w}}=\frac{\left(\mathbf{I}-\mathbf{U U}^{H}\right) \mathbf{s}}{\mathbf{s}^{H}\left(\mathbf{I}-\mathbf{U U}^{H}\right) \mathbf{s}},
$$

Or:

$$
\widehat{\mathbf{w}}=\mu \mathbf{U}\left(\widehat{\boldsymbol{\Gamma}}^{-1}+\frac{1}{\widehat{\sigma}_{n}^{2}} \mathbf{I}\right) \mathbf{U}^{H} \mathbf{s} .
$$

(In RD version, $\overline{\mathbf{U}}$ instead of $\mathbf{U}$ )

Algorithm 1: The proposed KA-STAP algorithm.

$N_{c}^{2} N M$ ). It should be also noted that the approach of the Gram-Schmidt orthogonalization can be applied to arbitrary arrays if we can obtain prior knowledge of the array geometry, some radar system parameters, and some information of the platform.

In particular, for the case of a side-looking ULA, we can further reduce the computational complexity to compute the clutter subspace eigenvectors. Since the dimension of the columns of $\mathbf{V}$ should satisfy $N_{c} \gg N_{r}$, if we carry out the Gram-Schmidt orthogonalization procedure on the columns 
of $\mathbf{V}$ one by one, this will result in unnecessary computations due to the linear correlation among the columns. Thus, it is desirable to directly find a group of vectors that are linear independent or nearly linear independent (i.e., most of the vectors are linearly independent and only very few vectors are linearly correlated). Fortunately, for a ULA we have the following proposition.

Proposition 1. For the case of a side-looking ULA and constant PRF, the clutter subspace belongs to the subspace computed by a group of space-time steering vectors $\left\{\overline{\mathbf{v}}_{p}\right\}_{p=0}^{N_{r}}$, which are given by

$$
\overline{\mathbf{v}}_{p}(n, m)=\exp \left(j 2 \pi f_{s}(\beta n+m)\right),
$$

where

$$
f_{s}=\frac{p}{N_{r}}, \quad p=0,1, \ldots, N_{r}-1
$$

Proof. Let us stack the above space-time steering vectors into a $N_{r} \times N M$ matrix $\widetilde{\mathbf{V}}$, which is shown as

$$
\widetilde{\mathbf{V}}=\left[\begin{array}{cccc}
1 & 1 & \cdots & 1 \\
z_{0,0} & z_{0,1} & \cdots & z_{N, M} \\
\vdots & \vdots & \vdots & \vdots \\
z_{0,0}^{N_{r}-1} & z_{0,1}^{N_{r}-1} & \cdots & z_{N, M}^{N_{r}-1}
\end{array}\right]
$$

where

$$
z_{n, m}=\exp \left(j 2 \pi \frac{\beta n+m}{N_{r}}\right) .
$$

Note that $\widetilde{\mathbf{V}}$ is a Vandermonde matrix of dimension $N_{r} \times N M$. For $z_{n, m}, n=0, \ldots, N-1$ and $m=0, \ldots, M-1$, the number of linearly independent columns of $\widetilde{\mathbf{V}}$ is determined by the number of distinct values of $\beta n+m$. If $\beta$ is an integer, the number of distinct values of $\beta n+m$ is $N_{r}=\beta(N-1)+M$. If $\beta$ is a rational value (not an integer), the number of distinct values of $\beta n+m$ is larger than $N_{r}=\lceil M+\beta(N-1)\rceil$. Therefore, $\widetilde{\mathbf{V}}$ has full rank, which is equal to [1]

$$
\operatorname{rank}(\widetilde{\mathbf{V}})=\min \left(N_{r}, N M\right)=N_{r} .
$$

The dimension of the clutter subspace is also $N_{r}$. Herein, the clutter subspace shares the same subspace with $\widetilde{\mathbf{V}}$. We can then compute the clutter subspace by taking the Gram-Schmidt orthogonalization procedure on the rows of $\widetilde{\mathbf{V}}$. Moreover, it should be noted that the computational complexity of the second approach is on the order of $O\left(\left(N_{r}+\right.\right.$ 1) $\left.N_{r} N M / 2\right) \ll O\left(\left(N_{c}+1\right) N_{c} N M / 2\right) \ll O\left(N_{c}^{3}+N_{c}^{2} N M\right)$, which exhibits a much lower complexity compared with the LSE resulting in a very useful tool for practical applications. It also avoids the procedure to determine the values of the number of clutter patches $N_{c}$ and the azimuth angle $\phi$.

After computing the orthogonal basis of the clutter subspace, we try to design the STAP filter weights by two different kinds of methods. One is to use the minimum norm eigen-canceler (MNE) derived in [5] to form the filter weights. Specifically, the MNE method is a linearly constrained beamformer with a minimum norm weight vector appearing orthogonal to the clutter subspace, which is described by [5]

$$
\begin{array}{ll}
\min _{\mathbf{w}} & \mathbf{w}^{H} \mathbf{w}, \\
\text { subject to } & \mathbf{U}^{H} \mathbf{w}=0, \quad \mathbf{w}^{H} \mathbf{s}=1 .
\end{array}
$$

The solution to the above optimization problem in (20) is provided by [5]

$$
\widehat{\mathbf{w}}=\frac{\left(\mathbf{I}-\mathbf{U U}^{H}\right) \mathbf{s}}{\mathbf{s}^{H}\left(\mathbf{I}-\mathbf{U} \mathbf{U}^{H}\right) \mathbf{s}} .
$$

The other method tries to design the filter weights using both the orthogonal bases of the computed clutter subspace and the observation data. Let us first calculate the root eigenvalues by projecting the data on the clutter subspace $\mathbf{U}$, as described by

$$
\widehat{\gamma}=\mathbf{U}^{H} \mathbf{x}
$$

Then, the clutter plus noise covariance matrix $\widehat{\mathbf{R}}$ can be estimated by

$$
\widehat{\mathbf{R}}=\mathbf{U} \widehat{\boldsymbol{\Gamma}} \mathbf{U}^{H}+\widehat{\sigma}_{n}^{2} \mathbf{I},
$$

where $\widehat{\Gamma}=\operatorname{diag}\left(\widehat{\gamma} \odot \widehat{\gamma}^{*}\right)$ and $\odot$ denotes the Hadamard product. It should be noted that, in (23), $\mathbf{U} \widehat{\boldsymbol{\Gamma}} \mathbf{U}^{H}$ is the estimated prior CCM. It introduces a linear combiner of the prior CCM and the sample covariance matrix (SCM) in [17, 24]. How to adaptively set the linear coefficients is also discussed in $[23,29]$. Observing $\widehat{\mathbf{R}}$ in (23), it corresponds to the case that the linear combiner of the prior CCM and the SCM with only thermal noise. Finally, the STAP filter weights can be computed by

$$
\widehat{\mathbf{w}}=\mu \mathbf{U}\left(\widehat{\boldsymbol{\Gamma}}^{-1}+\frac{1}{\widehat{\sigma}_{n}^{2}} \mathbf{I}\right) \mathbf{U}^{H} \mathbf{s},
$$

where we use the fact that $\widehat{\mathbf{R}}^{-1}=\mathbf{U}\left(\widehat{\boldsymbol{\Gamma}}^{-1}+\left(1 / \widehat{\sigma}_{n}^{2}\right) \mathbf{I}\right) \mathbf{U}^{H}$. The whole procedure of the proposed KA-STAP algorithm is summarized in Algorithm 1.

3.3. Proposed KA-STAP Employing CMT. In practice, there are many nonideal effects, such as the ICM and the channel mismatch [3], which result in performance degradation of the proposed algorithm. In the following, we will detail the proposed KA-STAP employing CMT.

For the angle-independent channel mismatch (since the main beam is usually fixed in a CPI, the angle-dependent channel mismatch can be approximated by spatial random tapers only related to the main beam and seen as angleindependent [3]), we can assume the spatial taper $\boldsymbol{\alpha}_{s}$ is a random vector but stable over a CPI due to the narrowband case considered in the paper [3]. Herein, the clutter plus noise 
received data vector in presence of channel mismatch is given by [3]

$$
\mathbf{x}=\left(\mathbf{V} \odot \Xi_{s}\right) \boldsymbol{\sigma}+\mathbf{n},
$$

where the columns of $\boldsymbol{\Xi}_{s}$ are all equivalent to $\mathbf{1}_{N} \otimes \boldsymbol{\alpha}_{s}$ and $\mathbf{1}_{N}$ denotes the all 1 vector with dimension $N$. When considering ICM, the received data can be represented as [3]

$$
\mathbf{x}=\left(\mathbf{V}_{s} \boldsymbol{\sigma}\right) \odot\left(\boldsymbol{\alpha}_{d} \otimes \mathbf{1}_{M}\right)+\mathbf{n},
$$

where $\mathbf{V}_{s}=\mathbf{V} \odot \boldsymbol{\Xi}_{s}$ and $\boldsymbol{\alpha}_{d}$ is the temporal taper accounting for the ICM. Then, the clutter plus noise covariance matrix is

$$
\mathbf{R}=\mathbf{R}_{s} \odot \mathbf{T}_{d}+\sigma_{n}^{2} \mathbf{I},
$$

where

$$
\begin{gathered}
\mathbf{R}_{s}=\mathbf{V}_{s} \boldsymbol{\Sigma} \mathbf{V}_{s}^{H}, \\
\mathbf{T}_{d}=E\left[\boldsymbol{\alpha}_{d} \boldsymbol{\alpha}_{d}^{H}\right] \otimes \mathbf{1}_{M, M},
\end{gathered}
$$

where $\mathbf{T}_{d}$ denotes the space-time CMT accounting for the ICM and $\mathbf{1}_{M, M}$ is the $M \times M$ all 1 matrix. One common model, referred to as the Billingsley model, is suitable for a land scenario. Its temporal autocorrelation is detailed in [3]. Another common model, presented by Ward in [1], is suitable for a water scenario. The temporal autocorrelation of the fluctuations is Gaussian in shape with the form:

$$
\zeta(m)=\exp \left\{-\frac{8 \pi^{2} \sigma_{v}^{2} T_{r}^{2}}{\lambda_{c}^{2}} m^{2}\right\},
$$

where $\sigma_{v}$ is the variance of the clutter spectral spread in $\mathrm{m}^{2} / \mathrm{s}^{2}$. Then, $\mathbf{T}_{d}$ can be given

$$
\mathbf{T}_{d}=\operatorname{Toeplitz}(\zeta(0) ; \ldots ; \zeta(N-1)) .
$$

In this paper, we consider the latter one.

Since $\boldsymbol{\alpha}_{d}$ is a random vector and its temporal autocorrelation has the form of (30), we assume the elements of $\boldsymbol{\alpha}_{d}$ do not equate to zeros, and we assume $\overline{\boldsymbol{\alpha}}_{d}=\left[1 / \alpha_{d, 1}, \ldots, 1 / \alpha_{d, N}\right]^{T}$. If we multiply both sides of (26) by $\overline{\boldsymbol{\alpha}}_{d} \otimes \mathbf{1}_{M}$, then it becomes

$$
\mathbf{x}_{s}=\mathbf{x} \odot\left(\boldsymbol{\alpha}_{d} \otimes \mathbf{1}_{M}\right)=\mathbf{V}_{s} \boldsymbol{\sigma}+\mathbf{n}_{s},
$$

where $\mathbf{n}_{s}=\mathbf{n} \odot\left(\boldsymbol{\alpha}_{d} \otimes \mathbf{1}_{M}\right)$. In this situation, similarly as the analysis in Section 3.2, we can employ the GramSchmidt orthogonalization procedure to compute a matrix with eigenvectors of $\mathbf{V}_{s}$, which is denoted as $\mathbf{U}_{s}$. Then the root eigenvalues $\gamma_{s}$ can be calculated by

$$
\gamma_{s}=\mathbf{U}_{s}^{H} \mathbf{x}_{s} .
$$

We can then compute $\mathbf{R}_{s}$ as

$$
\mathbf{R}_{s}=\mathbf{U}_{s} \boldsymbol{\Gamma}_{s} \mathbf{U}_{s}^{H},
$$

where

$$
\begin{aligned}
\boldsymbol{\Gamma}_{s} & =\operatorname{diag}\left(E\left[\boldsymbol{\gamma}_{s} \odot \boldsymbol{\gamma}_{s}^{*}\right]\right) \\
& =\operatorname{diag}\left(E\left[\left(\mathbf{U}_{s} \mathbf{x}_{s}\right) \odot\left(\mathbf{U}_{s} \mathbf{x}_{s}\right)^{*}\right]\right) \\
& =\operatorname{diag}\left(\left(\mathbf{U}_{s} \odot \mathbf{U}_{s}^{*}\right) E\left[\left(\mathbf{x}_{s} \odot \mathbf{x}_{s}^{*}\right)\right]\right) \\
& =\operatorname{diag}\left(\left(\mathbf{U}_{s} \odot \mathbf{U}_{s}^{*}\right) E\left[\left(\mathbf{x} \odot \mathbf{x}^{*}\right)\right]\right) \\
& =\operatorname{diag}\left(E\left[\left(\mathbf{U}_{s} \mathbf{x}\right) \odot\left(\mathbf{U}_{s} \mathbf{x}\right)^{*}\right]\right) .
\end{aligned}
$$

Here, it uses the fact that the expectation of the amplitude of the temporal taper caused by the ICM is one, which agrees with the ICM models introduced previously. From (34), we observe that $\mathbf{R}_{s}$ can be estimated using the received data $\mathbf{x}$ directly without $\boldsymbol{\alpha}_{d}$. It also suggested that the KAPE approach could directly use the received data and the calibrated spacetime steering vectors (only the spatial taper without the temporal taper) to compute the parameter $\boldsymbol{\sigma}$.

In order to obtain the clutter plus noise covariance matrix $\mathbf{R}$, we should estimate $\mathbf{R}_{s}$ and $\mathbf{T}_{d}$ in (27). Regarding the estimation of $\mathbf{R}_{s}$, we can firstly use the array calibration methods discussed in [19] to estimate the spatial taper (denoted as $\widehat{\boldsymbol{\alpha}}_{\boldsymbol{s}}$ ), which will not be discussed here due to space limitations. The reader is referred to the literature [19] for further details. Then, substituting $\widehat{\boldsymbol{\alpha}}_{s}$ into $\mathbf{V}_{s}$, we obtain the estimate $\widehat{\mathbf{V}}_{s}$ and then compute the eigenvector matrix $\widehat{\mathbf{U}}_{s}$. Finally, the estimate of $\widehat{\boldsymbol{\Gamma}}_{s}$ can be approximated using estimates obtained by the averages over the available snapshots instead of the expectation operation. Regarding the estimation of $\mathbf{T}_{d}$, it can be obtained via a rough knowledge of the interference environment (e.g., forest versus desert, bandwidth, etc.) [6].

After computing the estimates $\widehat{\mathbf{R}}_{s}$ and $\widehat{\mathbf{T}}_{d}$, we can compute the CCM as $\widehat{\mathbf{R}}_{c}=\widehat{\mathbf{R}}_{s} \odot \widehat{\mathbf{T}}_{d}$. Since $\widehat{\mathbf{R}}_{c}$ has low rank, we adopt the Lanczos algorithm to compute the clutter subspace $\mathbf{U}$, where the computational complexity is on the order of $O\left((N M)^{2} N_{r}^{\prime}\right)$ ( $N_{r}^{\prime}$ is the clutter rank of $\widehat{\mathbf{R}}_{c}$ ). Finally, the STAP filter weights are computed according to (21) or (24). The whole procedure can be seen in Algorithm 1.

Prior Knowledge Uncertainty Impact. In the proposed algorithms, prior knowledge uncertainty, such as velocity misalignment and yaw angle misalignment, will have a great impact on the performance. However, the scheme that employs the CMT will mitigate this impact. To illustrate this, we take a typical airborne radar system, for example. The parameters of the radar system are listed at the beginning of Section 4. Consider a far field scenario, the elevation angle will be close to zero resulting in $\cos \theta \approx 1$. Let $v_{p u}$ and $\phi_{u}$ denote the velocity deviation and the yaw angle deviation, respectively. Then, for a discretized azimuth angle $\phi$, the spatial frequency $f_{s}$ and Doppler frequency $f_{d}$ can be represented as

$$
\begin{gathered}
f_{s}=\frac{d_{a}}{\lambda_{c}} \sin \phi, \\
f_{d}=\frac{2\left(v_{p}+v_{p u}\right) T_{r}}{\lambda_{c}} \sin \left(\phi+\phi_{u}\right) .
\end{gathered}
$$

From (35), we see that prior knowledge uncertainty will affect the position and shape of the clutter ridge, which leads to the mismatch between the exact and the assumed spacetime steering vectors. Figure 2 provides a more direct way to illustrate the impact of prior knowledge uncertainty on the clutter ridge in the spatiotemporal plane. By employing a CMT, the clutter spectra will become wider along the clutter ridge in the figure including the exact clutter ridge. From this point of view, the impact of prior knowledge 


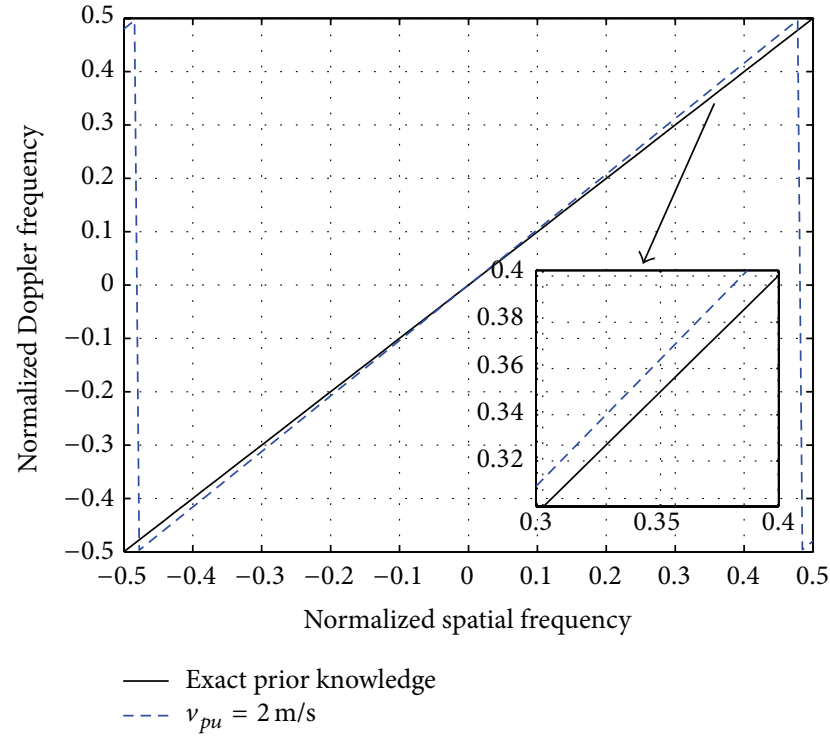

(a)

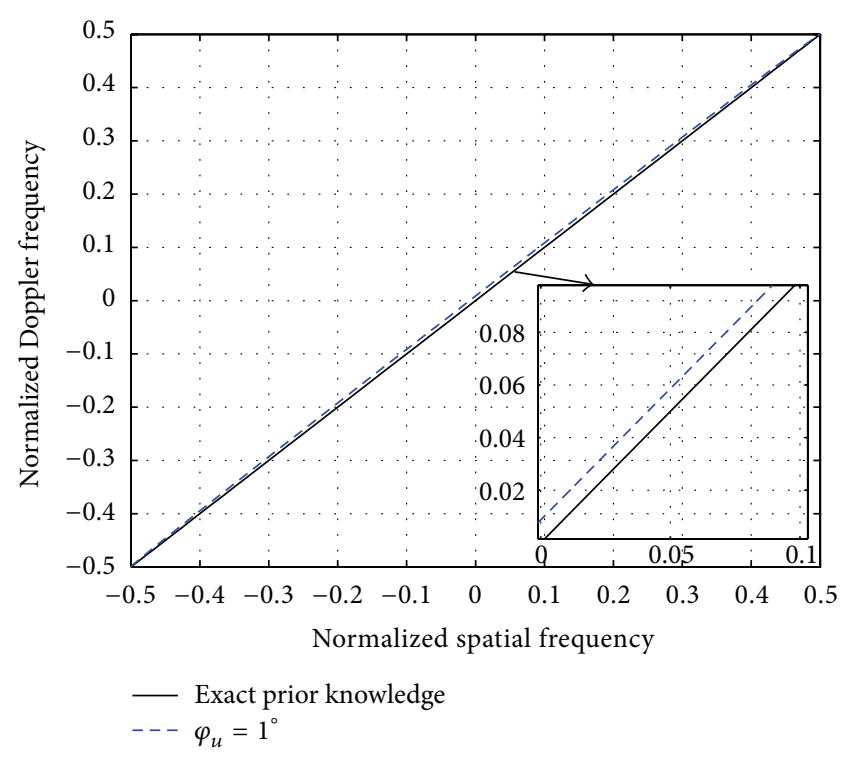

(b)

FIGURE 2: Impact of prior knowledge uncertainty to the clutter ridge in the spatiotemporal plane with (a) velocity deviation $v_{p u}=2 \mathrm{~m} / \mathrm{s}$ and (b) $\phi_{u}=1^{\circ}$.

uncertainty is mitigated. Because the methods in [20-22] do not consider any strategies to mitigate the impact of prior knowledge uncertainty, the performance will depend highly on the accuracy of prior knowledge. The KAPE approach in [19] also adopts the CMT and can mitigate the impact of prior knowledge uncertainty in a sense. But the differences between the proposed algorithm and the KAPE approach lie in three aspects. First, the KAPE approach estimates the CCM using the LS or some approximate approaches. On the contrary, the proposed algorithm estimates the CCM using the Gram-Schmidt orthogonalization procedure (that is not an approximation). Furthermore, for a side-looking ULA radar, the proposed algorithm directly selects a group of linearly independent space-time steering vectors using the LRGP and then takes the Gram-Schmidt orthogonalization procedure to compute the clutter subspace. Second, the proposed algorithm shows evidence that is feasible to directly use the received data vector and the calibrated space-time steering vectors (only the spatial taper without the temporal taper) to compute the parameter $\boldsymbol{\sigma}$. Third, the proposed algorithm with an $\mathrm{RD}$ version in the following section is presented to further reduce the complexity.

\subsection{Proposed Reduced-Dimension (RD) KA-STAP Algorithms.} From the above discussions, one aspect to be noted is that it is impractical to use all the DoFs available at the ULA for reasons of computational complexity when $N M$ is too large. In such situations, a common approach is to break the full DoFs problem into a number of smaller problems via the application of an $M N \times D$ (with $D \ll M N$ ) transformation matrix $\mathbf{S}_{D}$ to the data [1]. Our proposed KA-STAP algorithms can be easily extended to this kind of approach. By applying the reduced-dimension transformation matrix $\mathbf{S}_{D}$ to the data and the space-time steering vectors, we obtain

$$
\overline{\mathbf{x}}=\mathbf{S}_{D}^{H} \mathbf{x}, \quad \overline{\mathbf{V}}=\mathbf{S}_{D}^{H} \mathbf{V},
$$

where "-" denotes the results after the transformation. Then, the reduced-dimension CCM $\overline{\mathbf{R}}_{c}$ becomes

$$
\overline{\mathbf{R}}_{c}=\mathbf{S}_{D}^{H} \mathbf{R}_{c} \mathbf{S}_{D}=\overline{\mathbf{V}} \boldsymbol{\Sigma} \overline{\mathbf{V}}=\overline{\mathbf{U}} \bar{\Gamma} \overline{\mathbf{U}}^{H} .
$$

In a manner similar to that of the proposed full-DoF KASTAP algorithm described in Section 3.2, we compute the orthogonal bases of the clutter subspace $\overline{\mathbf{U}}$, estimate the CCM $\widehat{\overline{\mathbf{R}}}_{c}=\overline{\mathbf{U}} \hat{\bar{\Gamma}} \overline{\mathbf{U}}^{H}$, and then calculate the STAP filter weights according to (21) or (24). When employing a CMT to the ideal clutter covariance matrix, the final $\mathrm{RD}$ clutter covariance matrix can be estimated as

$$
\widehat{\overline{\mathbf{R}}}_{c}=\widehat{\overline{\mathbf{R}}}_{s} \odot \hat{\overline{\mathbf{T}}}_{d}=\left(\widehat{\overline{\mathbf{U}}}_{s} \hat{\overline{\boldsymbol{\Gamma}}}_{s} \widehat{\overline{\mathbf{U}}}_{s}^{H}\right) \odot \hat{\overline{\mathbf{T}}}_{d},
$$

where $\widehat{\overline{\mathbf{U}}}_{s}$ is computed by taking the Gram-Schmidt orthogonalization procedure to $\widehat{\overline{\mathbf{V}}}_{s}=\mathbf{S}_{D}^{H} \widehat{\mathbf{V}}_{s}, \widehat{\overline{\mathbf{\Gamma}}}_{s}$ is calculated via (34) using $\overline{\mathbf{x}}$ and $\widehat{\overline{\mathbf{U}}}_{s}$ instead of $\mathbf{x}$ and $\widehat{\mathbf{U}}_{s}$, and $\widehat{\overline{\mathbf{T}}}_{d}$ denotes the estimated RD CMT. Again, the STAP filter weights can be computed according to (21) or (24). By inspecting (37) and (38), we find that the computational complexity of our proposed RD-KA-STAP algorithm is related to $D$ instead of $N M(D \ll N M)$, which leads to great computation savings.

In this paper, we focus on the reduced-dimension technique known as extended factored (EFA) algorithm or multibin element-space post-Doppler STAP algorithm [1]. The simulations with this technique will show the performance of our proposed RD-KA-STAP algorithm. 


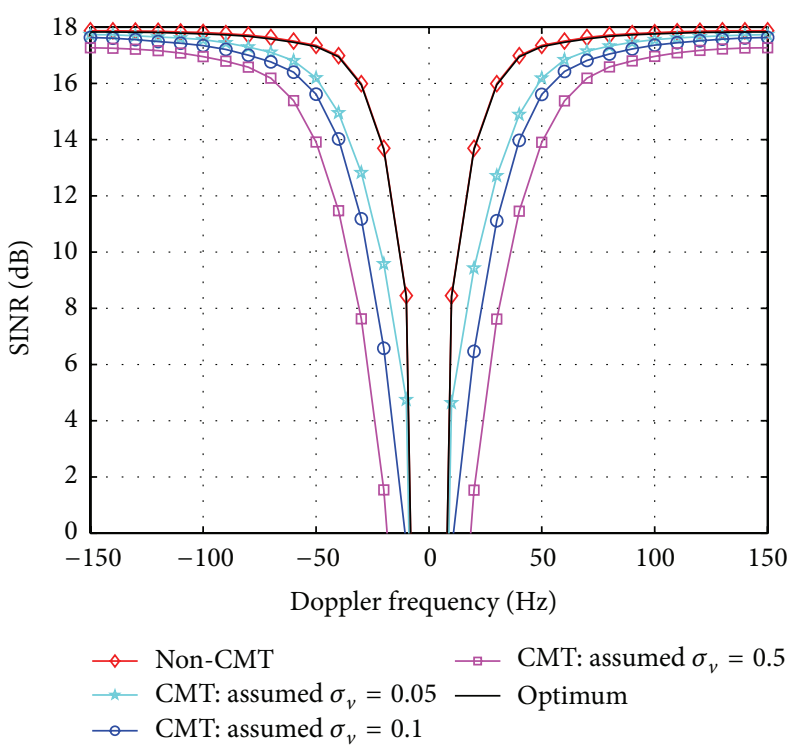

(a)

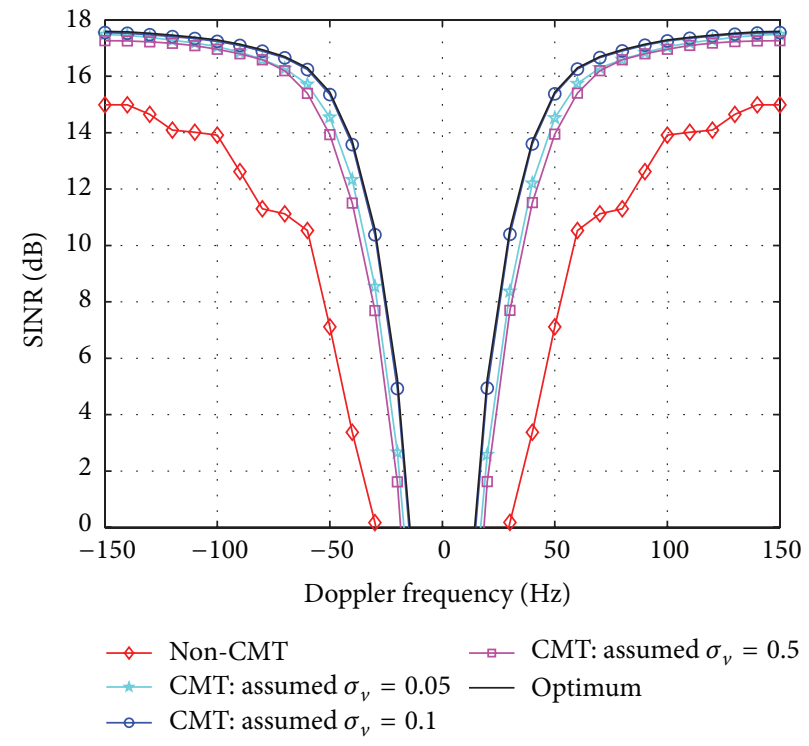

(b)

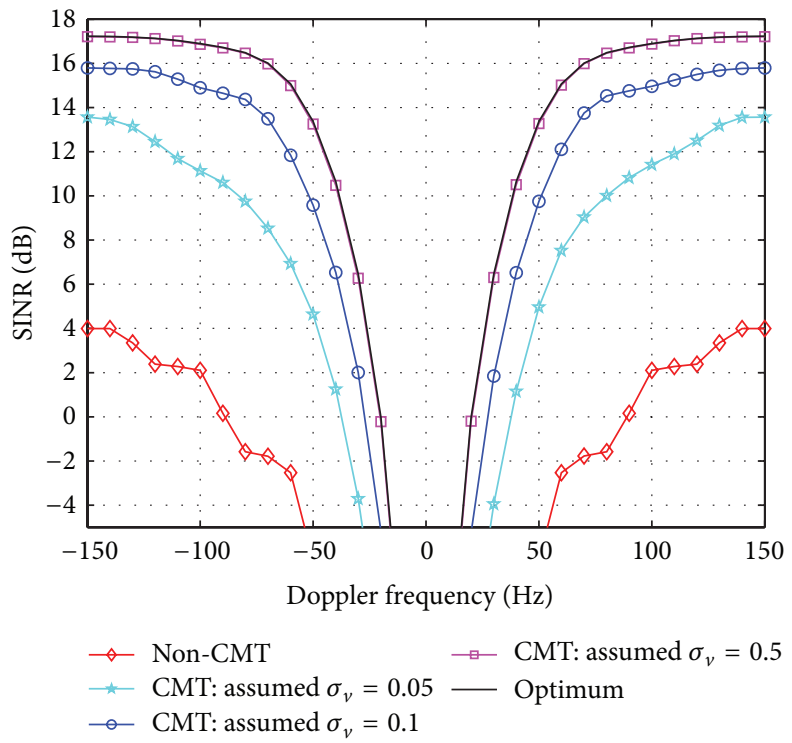

(c)

FIGURE 3: Impacts of ICM on SINR performance against Doppler frequency with 4 snapshots and the target Doppler frequency space from -150 to $150 \mathrm{~Hz}$. (a) $\sigma_{v}=0$; (b) $\sigma_{v}=0.1$; (c) $\sigma_{v}=0.5$.

3.5. Complexity Analysis. Here we illustrate the computational complexity of the proposed algorithms (shortened as LRGP KA-STAP and LRGP RD-KA-STAP) and other existing algorithms, namely, the sample matrix inversion algorithm (SMI), the EFA algorithm in [1], the joint-domain-localized (JDL) algorithm in [7], the CSMIECC algorithm in [20], and the KAPE algorithm in [19]. In Table 1, $D$ denotes the size of the reduced dimension. We can see that the computational complexity of our proposed algorithms is significantly lower than the CSMIECC and the KAPE algorithms $\left(N_{r} \ll\right.$ $\left.N_{c}, N M\right)$, which require the pseudoinverse of the matrix $\mathbf{V}^{H} \mathbf{V}$. With regard to the SMI algorithm, our proposed algorithms also show a lower computational complexity because the number of snapshots used for training the filter weights of the SMI is in the order of $2 N M$.

Although the computational complexity of the EFA and JDL algorithms is lower than our proposed LRGP KA-STAP algorithm, two aspects should be noted. One is that the number of snapshots used for training filter weights is much larger than our proposed algorithms. The other is that the computational complexity of EFA and JDL is proportional to the number of Doppler frequencies of interest (we only list the computation complexity for one Doppler frequency), while our proposed algorithms only have to compute the CCM once 
TABLE 1: Computational complexity of algorithms.

\begin{tabular}{lcc}
\hline Algorithm & Estimate the CCM & Compute filter weights \\
\hline SMI & $O\left(L(N M)^{2}\right)$ & $O\left((N M)^{3}\right)$ \\
\hline EFA & $O\left(L(D)^{2}\right)+O\left(L \frac{N}{2} \log _{2}(N)\right)$ & $O\left(D^{3}\right)$ \\
\hline JDL & $O\left(L(D)^{2}\right)+O\left(L \frac{N M}{2} \log _{2}(N M)\right)$ & $O\left(D^{3}\right)$ \\
\hline CSMIECC & $O\left(L(N M)^{2}\right)+O\left(N_{c}(N M)^{2}\right)+O\left(N_{c}^{3}+N_{c}^{2} N M\right)$ & $O\left((N M)^{3}\right)$ \\
\hline KAPE & $O\left(N_{c}(N M)^{2}\right)+O\left(N_{c}^{3}+N_{c}^{2} N M\right)$ & $O\left((N M)^{3}\right)$ \\
\hline LRGP KA-STAP & $O\left(\frac{\left(N_{r}+1\right) N_{r} N M}{2}+L N_{r} N M\right)+O\left(N_{r}(N M)^{2}\right)$ & $O\left(N_{r}(N M)^{2}\right)$ \\
\hline LRGP RD-KA-STAP & $O\left(\frac{\left(N_{r}+1\right) N_{r} D}{2}+L N_{r} D\right)+O\left(N_{r} D^{2}\right)$ & $O\left(D^{3}\right)$
\end{tabular}

for different Doppler frequencies of interest. Moreover, the computational complexity of our proposed LRGP RD-KASTAP is lower than the EFA since $L$ in EFA is in the order of $2 D$, where $D$ is usually larger than $N_{r}$.

\section{Performance Assessment}

In this section, we assess the proposed KA-STAP algorithms by computing the output SINR performance and probability of detection performance using simulated radar data. The output SINR is defined by

$$
\operatorname{SINR}=\frac{\left|\widehat{\mathbf{w}}^{H} \mathbf{s}\right|^{2}}{\left|\widehat{\mathbf{w}}^{H} \mathbf{R} \widehat{\mathbf{w}}\right|} .
$$

Throughout the simulations, unless otherwise stated, the simulated scenarios use the following parameters: side-looking ULA, uniform transmit pattern, $M=8, N=8, f_{c}=$ $450 \mathrm{MHz}, f_{r}=300 \mathrm{~Hz}, v_{p}=50 \mathrm{~m} / \mathrm{s}, d_{a}=\lambda_{c} / 2, \beta=1$, $N_{r}=\lceil M+\beta(N-1)\rceil=15, h_{p}=9000 \mathrm{~m}$, signal-to-noise ratio (SNR) of $0 \mathrm{~dB}$, the target located at $0^{\circ}$ azimuth with Doppler frequency $100 \mathrm{~Hz}$, clutter-to-noise ratio (CNR) of $50 \mathrm{~dB}$, and unitary thermal noise power (here, SNR and CNR are defined relative to noise power per channel and per pulse). All presented results are averaged over 100 independent Monte Carlo runs.

4.1. Impact of ICM on the SINR Performance. In this subsection, we evaluate the impact on the SINR performance with different ICM for our proposed algorithms. In the examples, we consider four different ICM cases with $\sigma_{v}=0, \sigma_{v}=0.1$, and $\sigma_{v}=0.5$. The number of snapshots for training is 4 . In Figures 3(a), 3(b), and 3(c), we show the SINR performance against the target Doppler frequency of our proposed LRGP KA-STAP algorithm both with and without a CMT. From the figures, we observe the following conclusions. (i) When there is non-ICM, the proposed LRGP KA-STAP algorithm without a CMT can obtain the optimum performance since the computed clutter subspace is exact. However, it degrades the SINR performance with the increase of $\sigma_{v}$ resulting in extra sensitivity to the ICM. That is because the computed clutter subspace cannot represent the true clutter subspace. (ii) Our proposed LRGP KA-STAP algorithm with a CMT illustrates a robust characteristic to the ICM. When the estimated parameter $\sigma_{v}$ of CMT is correct, we can achieve the optimum SINR performance. Furthermore, the range of values of CMT mismatch is demonstrated in which the estimated spreading exhibits acceptable SINR performance, which can be useful in applications. This can be interpreted as that the computed clutter subspace via the application of the CMT to the ideal clutter subspace spans a similar space to the true clutter subspace.

4.2. Impact of Inaccurate Prior Knowledge on the SINR Performance. In this subsection, we focus on the impact of inaccurate prior knowledge on the SINR performance of our proposed algorithms. In the first example, we consider the impact of the velocity misalignment by showing the SINR performance against the target Doppler frequency, as shown in Figure 4. Consider three different cases: the velocity misalignments of prior knowledge are (a) $0.5 \mathrm{~m} / \mathrm{s}$, (b) $1 \mathrm{~m} / \mathrm{s}$, and (c) $2 \mathrm{~m} / \mathrm{s}$, compared with true platform velocity. The potential Doppler frequency space from -150 to $150 \mathrm{~Hz}$ is examined and 4 snapshots are used to train the filter weights. The plots show that the proposed LRGP KA-STAP algorithm without a CMT is sensitive to the velocity misalignment, while the LRGP KA-STAP algorithm with a CMT is robust to that. The reason for this is that the velocity misalignment of prior knowledge will lead to the mismatch between the computed clutter subspace and the true clutter subspace. Although the computed clutter subspace via the CMT cannot avoid this situation, it can mitigate this impact. Because the velocity misalignment between the clutter patches and the platform can be seen as the Doppler spreading of the clutter patches. Moreover, the results also show that a slightly larger value of the estimated parameter $\sigma_{v}$ will result in an improved SINR performance for the velocity misalignment case.

The evaluation of the impact caused by the yaw angle misalignment is shown in Figure 5, where we also consider three different cases: the yaw angle misalignments of prior knowledge are (a) $0.2^{\circ}$, (b) $0.5^{\circ}$, and (c) $1^{\circ}$. The curves also indicate that (i) the proposed LRGP KA-STAP algorithm without a CMT is sensitive to the yaw angle misalignment, while the LRGP KA-STAP algorithm with a CMT is robust 


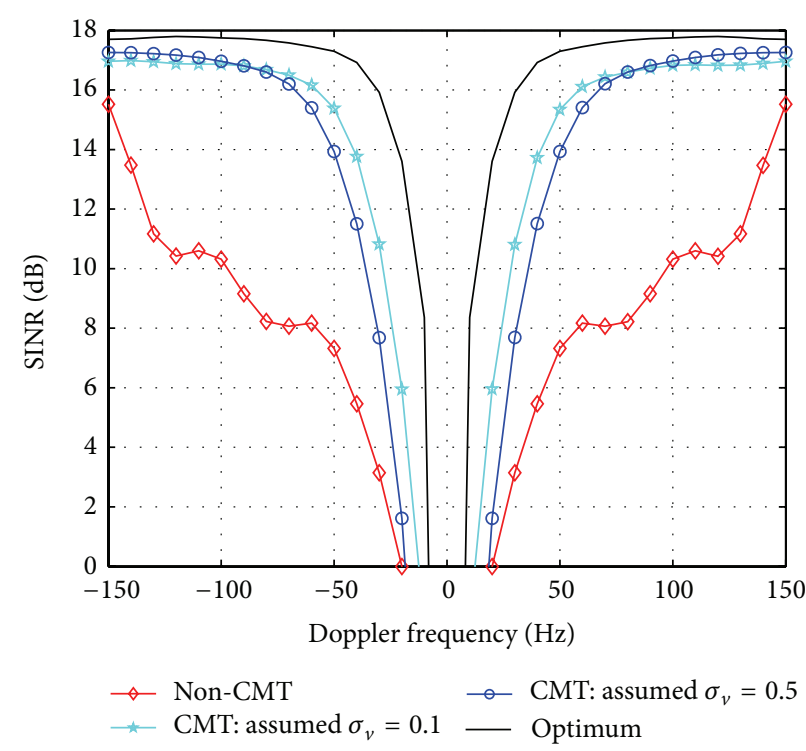

(a)

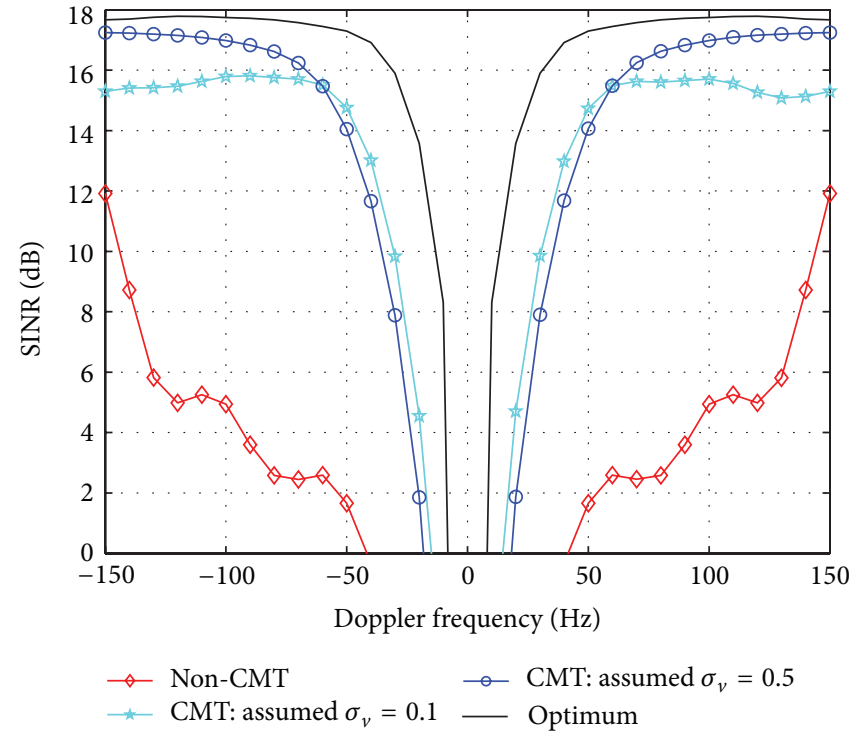

(b)

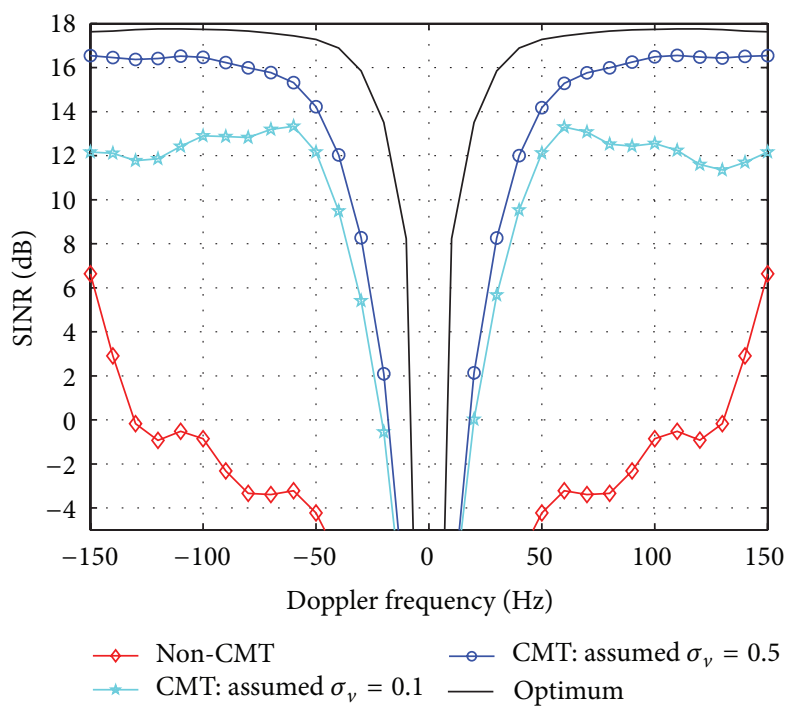

(c)

FIGURE 4: Impacts of velocity misalignment of prior knowledge on SINR performance against Doppler frequency with 4 snapshots and the target Doppler frequency space from -150 to $150 \mathrm{~Hz}$. (a) Velocity misalignment $0.5 \mathrm{~m} / \mathrm{s}$; (b) velocity misalignment $1 \mathrm{~m} / \mathrm{s}$; (c) velocity misalignment $2 \mathrm{~m} / \mathrm{s}$.

to that; (ii) a slightly larger value of the estimated parameter $\sigma_{v}$ will result in an improved SINR performance. The misalignment of the yaw angle will lead to a Doppler frequency mismatch between the radar platform and the clutter patches. While the CMT mainly aims at mitigating the performance degradation caused by the clutter Doppler spreading, the CMT will lead to an improved estimated clutter subspace and will exhibit robustness against the yaw angle misalignment.

4.3. Comparison with Conventional STAP Algorithms. To provide further investigation about the performance of our proposed algorithms, we compare the SINR performance versus the snapshots of our proposed LRGP KA-STAP and LRGP
RD-KA-STAP algorithms with the Loaded SMI (LSMI), the EFA algorithm (3 Doppler bins), the $3 \times 3$ JDL algorithm, Stoica's scheme in [23] (the prior knowledge covariance matrix is computed in the same way as the CSMIECC algorithm), and the CSMIECC algorithm (the combination parameter is set to 0.6 ) in [20], where the simulation results are shown in Figure 6. Here, we consider a scenario of ICM with $\sigma_{v}=0.5$ and assume the diagonal loading factors for all algorithms are set to the level of the thermal noise power. The parameter $\sigma_{v}$ for our proposed algorithms is assumed to be 1 . The curves in the figure illustrate that our proposed algorithms have a very fast SINR convergence speed, which only needs three snapshots for training, and offer significant 


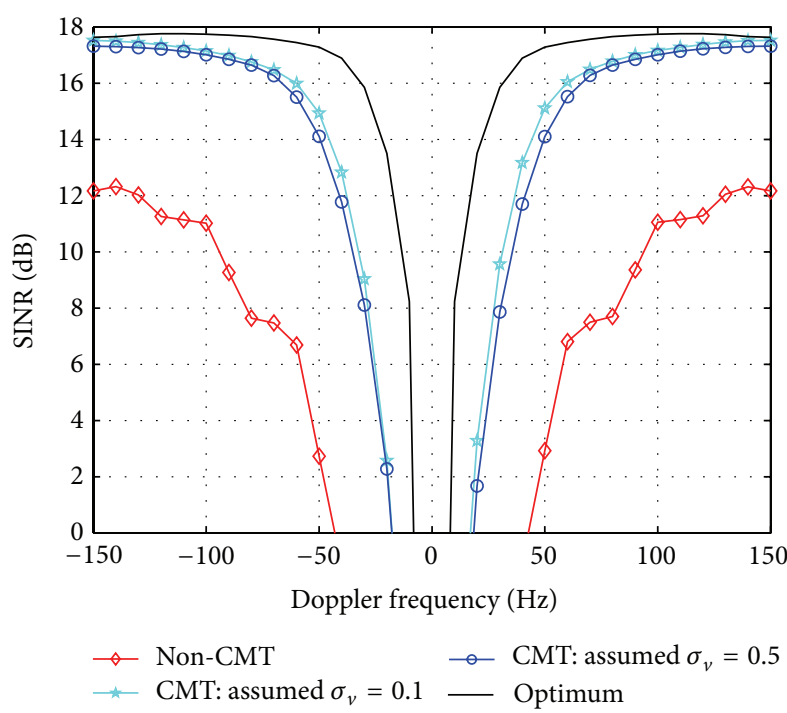

(a)

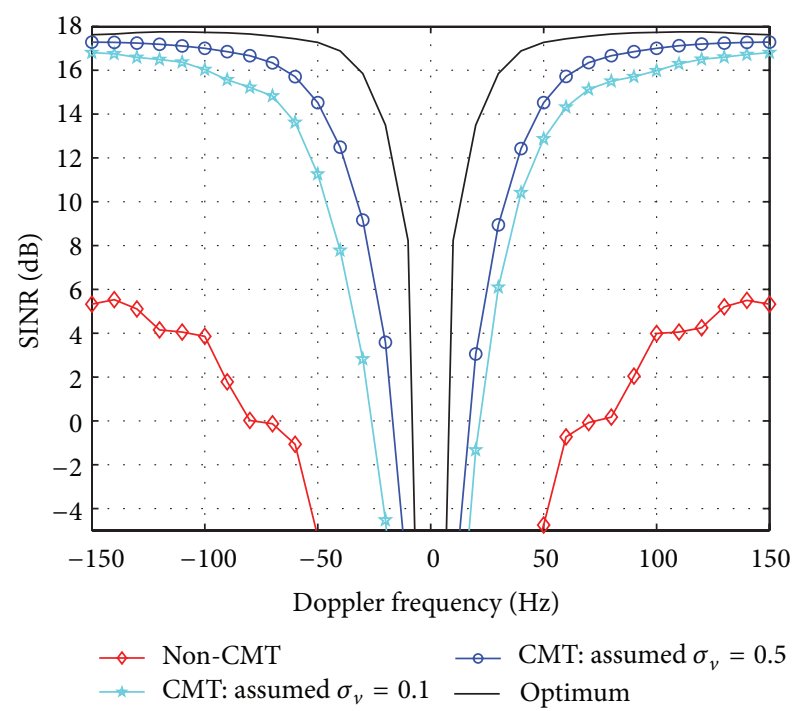

(b)

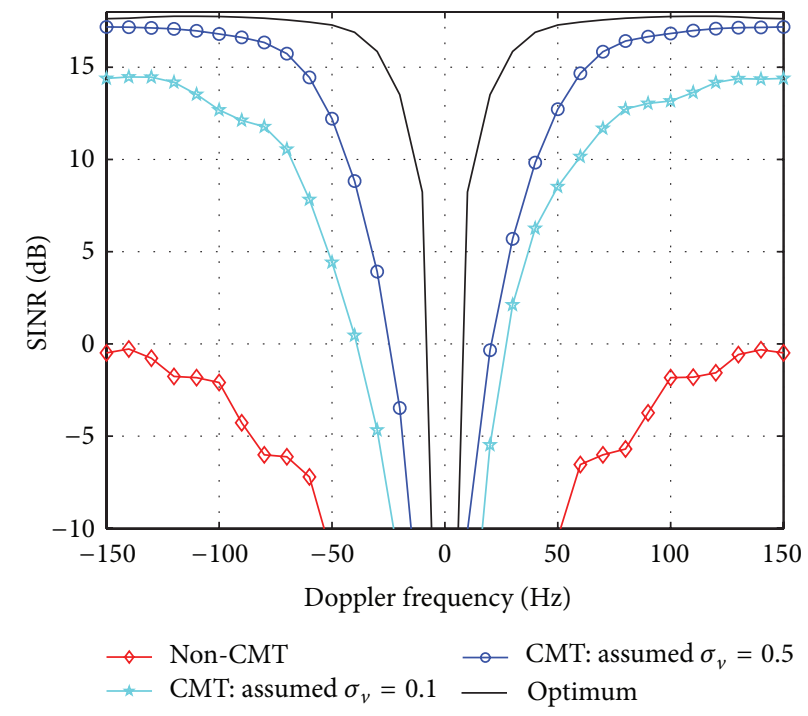

(c)

FIGURE 5: Impacts of yaw angle misalignment of prior knowledge on SINR performance against Doppler frequency with 4 snapshots and the target Doppler frequency space from -150 to $150 \mathrm{~Hz}$ : (a) yaw angle misalignment $0.2^{\circ}$, (b) yaw angle misalignment $0.5^{\circ}$, and (c) yaw angle misalignment $1^{\circ}$.

better SINR steady-state performance compared with the LSMI, EFA, JDL, Stoica's scheme, and CSMIECC algorithms. This is because the proposed algorithms provide a much better estimation of the CCM by using prior knowledge of the data, the low clutter rank property, the geometry of the array, and the interference environment. It should be noted that the SINR performance of the LRGP RD-KA-STAP algorithm is worse than that of LRGP KA-STAP with fullDOFs. This is due to the fact that the reduced DOFs will lead to lower computational complexity at the cost of performance degradation.

The results in Figure 7 illustrate the SINR performance versus the target Doppler frequency.The number of snapshots used for training in the LSMI, EFA, JDL, Stoica's scheme, and CSMIECC algorithms is set to 48 , while we set it to 4 in our proposed algorithms. It is found that our proposed LRGP KA-STAP algorithm provides the best SINR performance among all algorithms and forms the narrowest clutter null resulting in improved performance for the detection of slow targets. It is also shown that the performance of the proposed LRGP RD-KA-STAP algorithm is worse than that of LRGP KA-STAP with full-DOFs but better than other algorithms in most Doppler bins. Note that although the LRGP RD-KA-STAP algorithm performs slightly worse than other algorithms in Doppler range of -60 to $60 \mathrm{~Hz}$, it requires much less snapshots for training filter weights. 


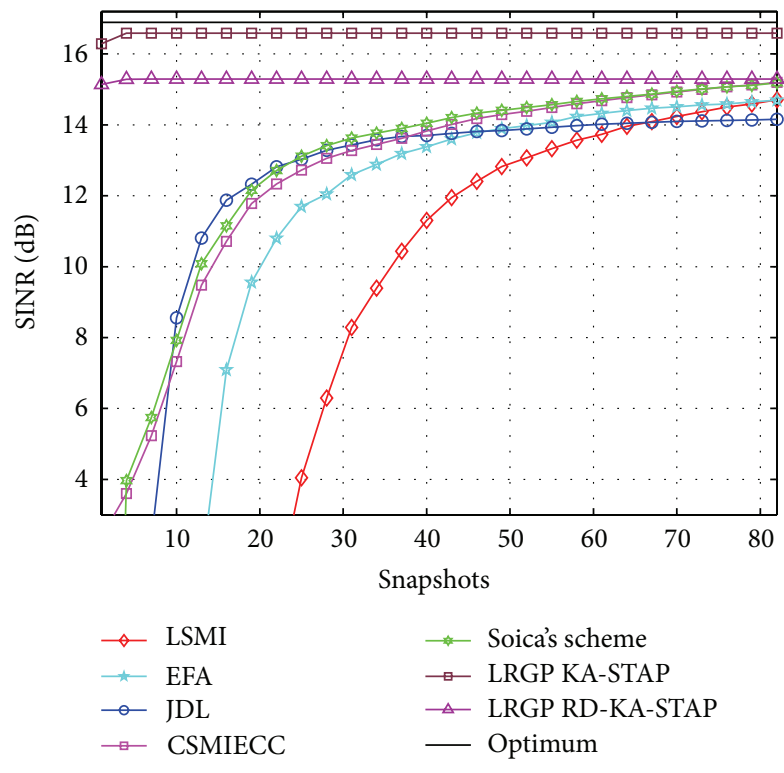

FIGURE 6: SINR performance against the number of snapshots considering ICM, where $\sigma_{v}=0.5$.

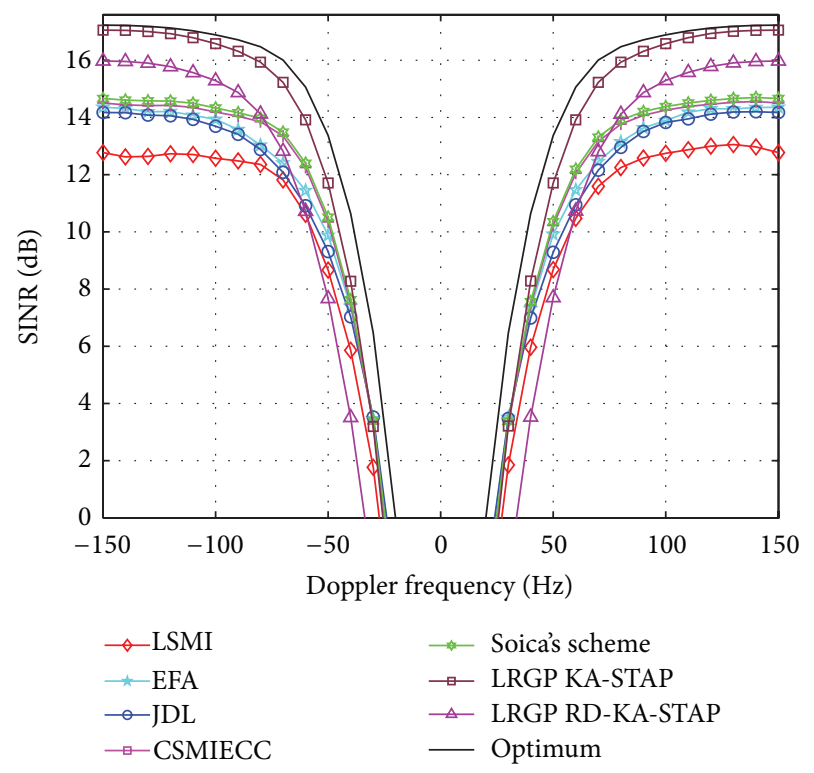

FIGURE 7: SINR performance versus the target Doppler frequency. The number of snapshots used for training in the LSMI, EFA, JDL, and CSMIECC algorithms is set to 48 , while we only use 4 snapshots for our proposed algorithms.

As shown in Figure 8, we present the probability of detection performance versus the target SNR for all algorithms. The false alarm rate is set to $10^{-3}$ and for simulation purposes the threshold and probability of detection estimates are based on 10,000 samples. We suppose the target is injected in the the boresight with Doppler frequency $100 \mathrm{~Hz}$. We note that the proposed algorithms provide suboptimal detection performance using very short snapshots but, remarkably,

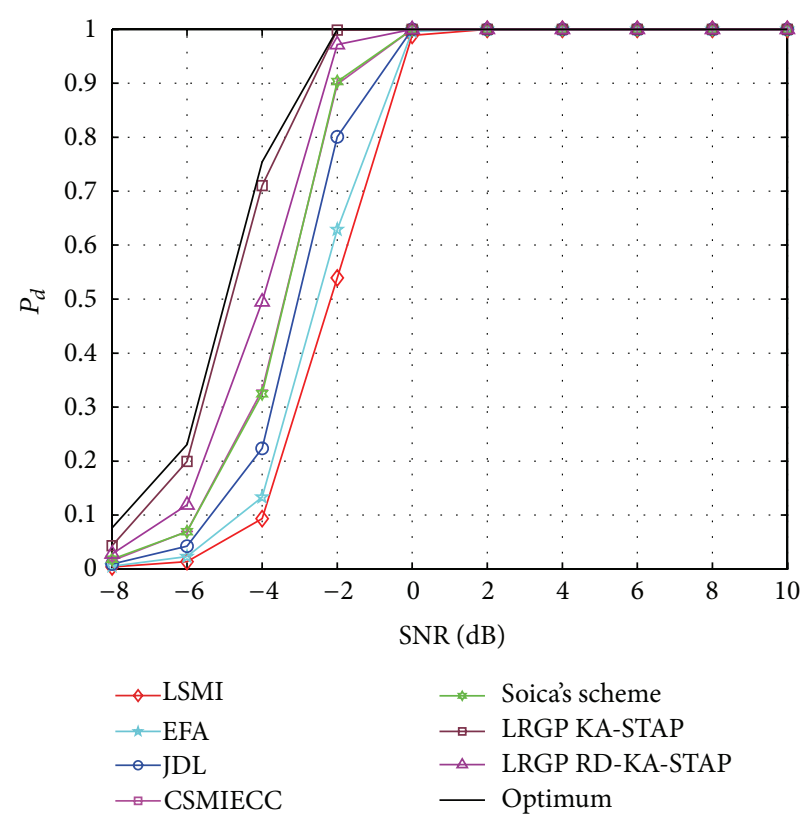

FIGURE 8: Probability of detection performance against the target SNR. Suppose the target is injected in the boresight with Doppler frequency $100 \mathrm{~Hz}$, and other parameters setting for all algorithms are the same as that in the second example.

obtain much higher detection rate than other algorithms at an SNR level from $-8 \mathrm{~dB}$ to $0 \mathrm{~dB}$.

\section{Conclusions}

In this paper, novel KA-STAP algorithms have been proposed by using prior knowledge of LRGP to obtain an accurate estimation of the CCM with a very small number of snapshots. By exploiting the fact that the clutter subspace is only determined by the space-time steering vectors, we have developed a Gram-Schmidt orthogonalization approach to compute the clutter subspace. In particular, for a sidelooking ULA, we have proposed a scheme to directly select a group of linearly independent space-time steering vectors to compute the orthogonal bases of the clutter subspace. Compared with the LSE algorithm, it has not only exhibited a low complexity, but also shown a simple way to compute the CCM. To overcome the performance degradation caused by the nonideal effects and prior knowledge uncertainty, the proposed KA-STAP algorithm that combines the CMT has been presented and a reduced-dimension version has been devised for practical applications. This has also provided evidence that is feasible to directly use the received data vector and the calibrated space-time steering vectors (only the spatial taper without the temporal taper) to compute the assumed clutter amplitude. The simulation results have shown that our proposed algorithms outperform other existing algorithms in terms of SINR steady-state performance, SINR convergence speed, and detection performance for a very small number of snapshots and also exhibit robustness against errors in prior knowledge. 


\section{Conflict of Interests}

The authors declare that there is no conflict of interests regarding the publication of this paper.

\section{Acknowledgments}

This work is funded in part by the National Natural Science Foundation of China $(61201334,61101182$, and 61171133) and the Hunan Provincial Innovation Foundation for Postgraduate.

\section{References}

[1] J. Ward, "Space-time adaptive processing for airborne radar," Tech. Rep. 1015, MIT Lincoln Laboratory, Lexington, Mass, USA, 1994.

[2] R. Klemm, Principles of Space-Time Adaptive Processing, Institute of Electical Engineering, London, UK, 2006.

[3] J. R. Guerci, Space-time Adaptive Processing for Radar, Artech House, 2003.

[4] W. L. Melvin, "A STAP overview," IEEE Aerospace and Electronic Systems Magazine, vol. 19, no. 1, pp. 19-35, 2004.

[5] A. M. Haemovich and M. Berin, "Eigenanalysis-based spacetime adaptive radar: performance analysis," IEEE Transactions on Aerospace and Electronic Systems, vol. 33, no. 4, pp. 1170-1179, 1997.

[6] J. R. Guerci and J. S. Bergin, "Principal components, covariance matrix tapers, and the subspace leakage problem," IEEE Transactions on Aerospace and Electronic Systems, vol. 38, no. 1, pp. 152-162, 2002.

[7] H. Wang and L. Cai, "On adaptive spatial-temporal processing for airborne surveillance radar systems," IEEE Transactions on Aerospace and Electronic Systems, vol. 30, no. 3, pp. 660-670, 1994.

[8] R. C. de Lamare, L. Wang, and R. Fa, "Adaptive reducedrank LCMV beamforming algorithms based on joint iterative optimization of filters: Design and analysis," Signal Processing, vol. 90, no. 2, pp. 640-652, 2010.

[9] R. Fa, R. C. de Lamare, and L. Wang, "Reduced-rank STAP schemes for airborne radar based on switched joint interpolation, decimation and filtering algorithm," IEEE Transactions on Signal Processing, vol. 58, no. 8, pp. 4182-4194, 2010.

[10] R. Fa and R. C. de Lamare, "Reduced-rank STAP algorithms using joint iterative optimization of filters," IEEE Transactions on Aerospace and Electronic Systems, vol. 47, no. 3, pp. 16681684, 2011.

[11] J. R. Román, M. Rangaswamy, D. W. Davis, Q. Zhang, B. Himed, and J. H. Michels, "Parametric adaptive matched filter for airborne radar applications," IEEE Transactions on Aerospace and Electronic Systems, vol. 36, no. 2, pp. 677-692, 2000.

[12] Z. Yang, R. C. de Lamare, and X. Li, " $L_{1}$-regularized STAP algorithms with a generalized sidelobe canceler architecture for airborne radar," IEEE Transactions on Signal Processing, vol. 60, no. 2, pp. 674-686, 2012.

[13] Z. Yang, R. C. de Lamare, and X. Li, "Sparsity-aware space-time adaptive processing algorithms with $L_{1}$-norm regularisation for airborne radar," IET Signal Processing, vol. 6, no. 5, pp. 413-423, 2012.

[14] Z. Yang, X. Li, H. Wang, and W. Jiang, "On clutter sparsity analysis in space-time adaptive processing airborne radar," IEEE
Geoscience and Remote Sensing Letters, vol. 10, no. 5, pp. 12141218, 2013.

[15] Z. Yang, X. Li, H. Wang, and W. Jiang, "Adaptive clutter suppression based on iterative adaptive approach for airborne radar," Signal Processing, vol. 93, no. 12, pp. 3567-3577, 2013.

[16] Z. Yang, X. Li, H. Wang, and L. Nie, "Sparsity-based space-time adaptive processing using complex-valued homotopy technique for airborne radar," IET Signal Processing, vol. 8, no. 5, pp. 552564, 2014.

[17] J. R. Guerci and E. J. Baranoski, "Knowledge-aided adaptive radar at DARPA," IEEE Signal Processing Magazine, vol. 23, no. 1, pp. 41-50, 2006.

[18] M. C. Wicks, M. Rangaswamy, R. Adve, and T. B. Hale, "Spacetime adaptive processing," IEEE Signal Processing Magazine, vol. 23, no. 1, pp. 51-65, 2006.

[19] W. L. Mevin and G. A. Showman, "An approach to knowledgeaided covariance estimation," IEEE Transactions on Aerospace and Electronic Systems, vol. 42, no. 3, pp. 1021-1042, 2006.

[20] W. Xie, K. Duan, F. Gao, Y. Wang, and Z. Zhang, "Clutter suppression for airborne phased radar with conformal arrays by least squares estimation," Signal Processing, vol. 91, no. 7, pp. 1665-1669, 2011.

[21] I. W. Selesnick, S. U. Pillai, K. Y. Li, and B. Himed, "AngleDoppler processing using sparse regularization," in Proceedings of the IEEE International Conference on Acoustics, Speech, and Signal Processing (ICASSP '10), pp. 2750-2753, March 2010.

[22] C. Chen and P. P. Vaidyanathan, "MIMO radar space-time adaptive processing using prolate spheroidal wave functions," IEEE Transactions on Signal Processing, vol. 56, no. 2, pp. 623635, 2008.

[23] P. Stoica, J. Li, X. Zhu, and J. Guerci, "On using a priori knowledge in space-time adaptive processing," IEEE Transactions on Signal Processing, vol. 56, no. 6, pp. 2598-2602, 2008.

[24] J. R. Guerci, Cognitive Radar: The Knowledge-Aided Fully Adaptive Approach, Artech House, London, UK, 2010.

[25] J. S. Bergin, P. M. Techau, W. L. Melvin, and J. R. Guerci, "GMTI STAP in target-rich environments: site-specific analysis," in Proceedings of the IEEE Radar Conference, pp. 391-396, Long Beach, Calif, USA, April 2002.

[26] J. S. Bergin, C. M. Teixeira, P. M. Techau, and J. R. Guerci, "Improved clutter mitigation performance using knowledgeaided space-time adaptive processing," IEEE Transactions on Aerospace and Electronic Systems, vol. 42, no. 3, pp. 997-1008, 2006.

[27] E. Conte, A. de Maio, A. Farina, and G. Foglia, "Design and analysis of a knowledge-aided radar detector for Doppler processing," IEEE Transactions on Aerospace and Electronic Systems, vol. 42, no. 3, pp. 1058-1079, 2006.

[28] K. Gerlach and M. L. Picciolo, "Airborne/spacebased radar STAP using a structured covariance matrix," IEEE Transactions on Aerospace and Electronic Systems, vol. 39, no. 1, pp. 269-281, 2003.

[29] X. Zhu, J. Li, and P. Stoica, "Knowledge-aided space-time adaptive processing," IEEE Transactions on Aerospace and Electronic Systems, vol. 47, no. 2, pp. 1325-1336, 2011.

[30] R. Fa, R. C. de Lamare, and V. H. Nascimento, "Knowledgeaided STAP algorithm using convex combination of inverse covariance matrices for heterogenous clutter," in Proceeding of the IEEE International Conference on Acoustics, Speech, and Signal Processing (ICASSP '10), pp. 2742-2745, Dallas, Tex, USA, March 2010. 
[31] Q. Zhang and W. B. Mikhael, "Estimation of the clutter rank in the case of subarraying for space-time adaptive processing," Electronics Letters, vol. 33, no. 5, pp. 419-420, 1997.

[32] N. A. Goodman and J. M. Stiles, "On clutter rank observed by arbitrary arrays," IEEE Transactions on Signal Processing, vol. 55, no. 1, pp. 178-186, 2007.

[33] R. A. Horn and C. R. Johnson, Matrix Analysis, Cambridge University Press, Cambridge, UK, 1985. 

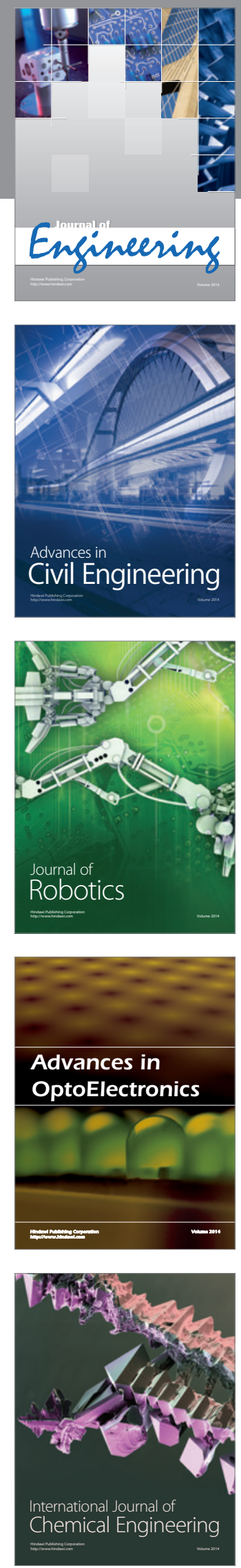

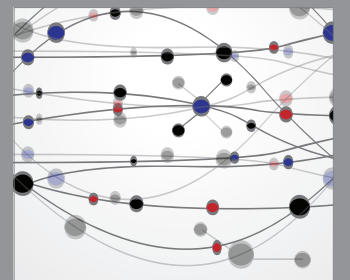

The Scientific World Journal
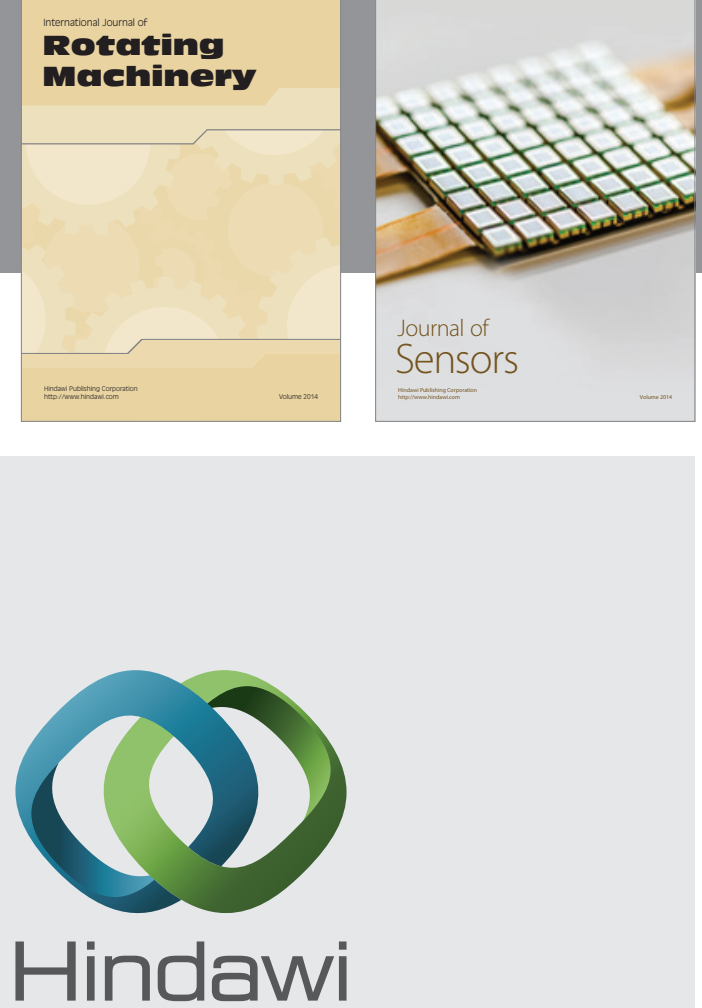

Submit your manuscripts at http://www.hindawi.com
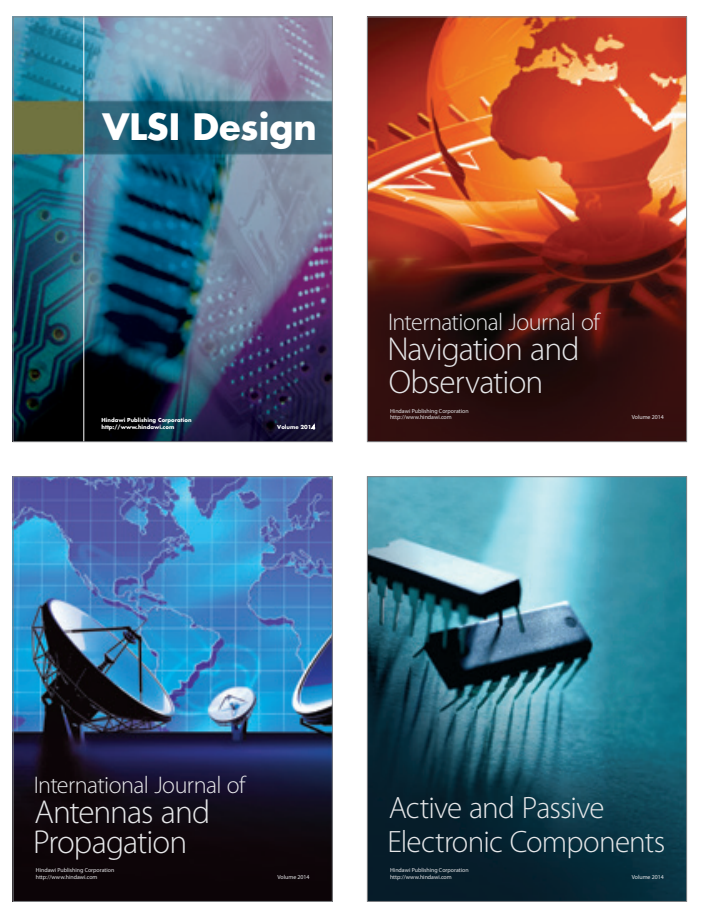
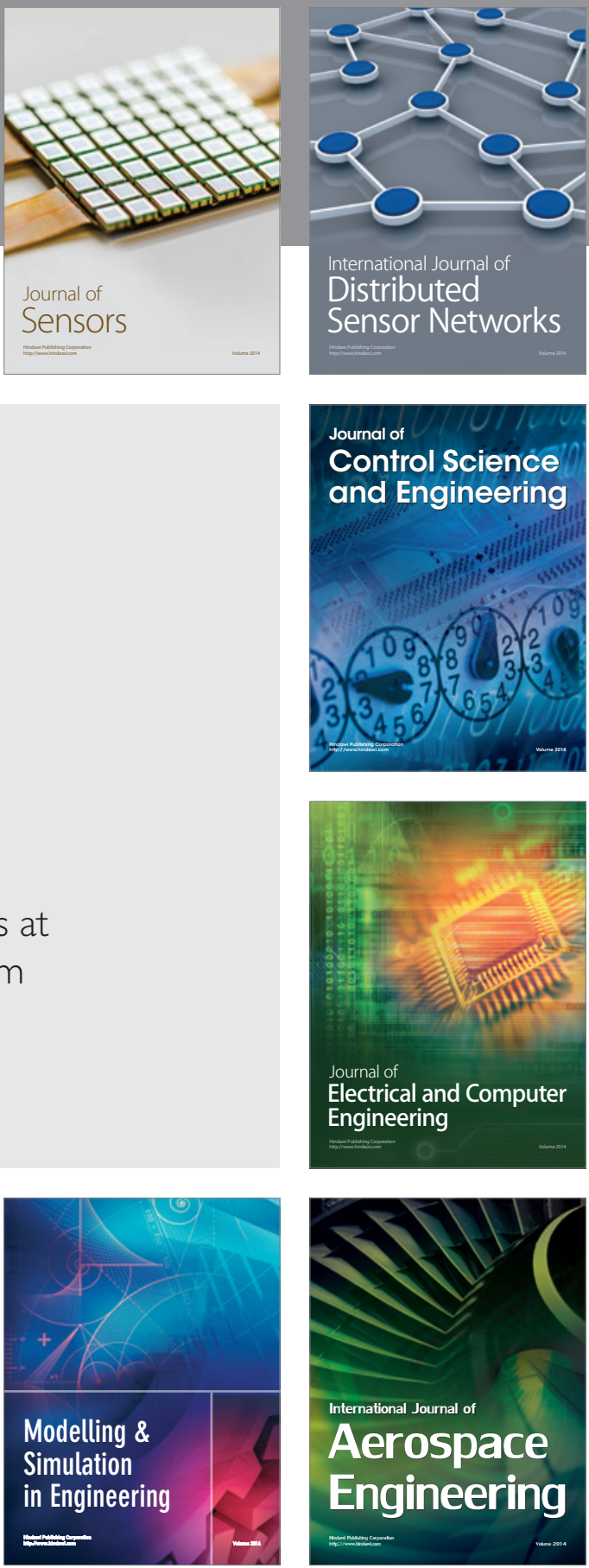

Journal of

Control Science

and Engineering
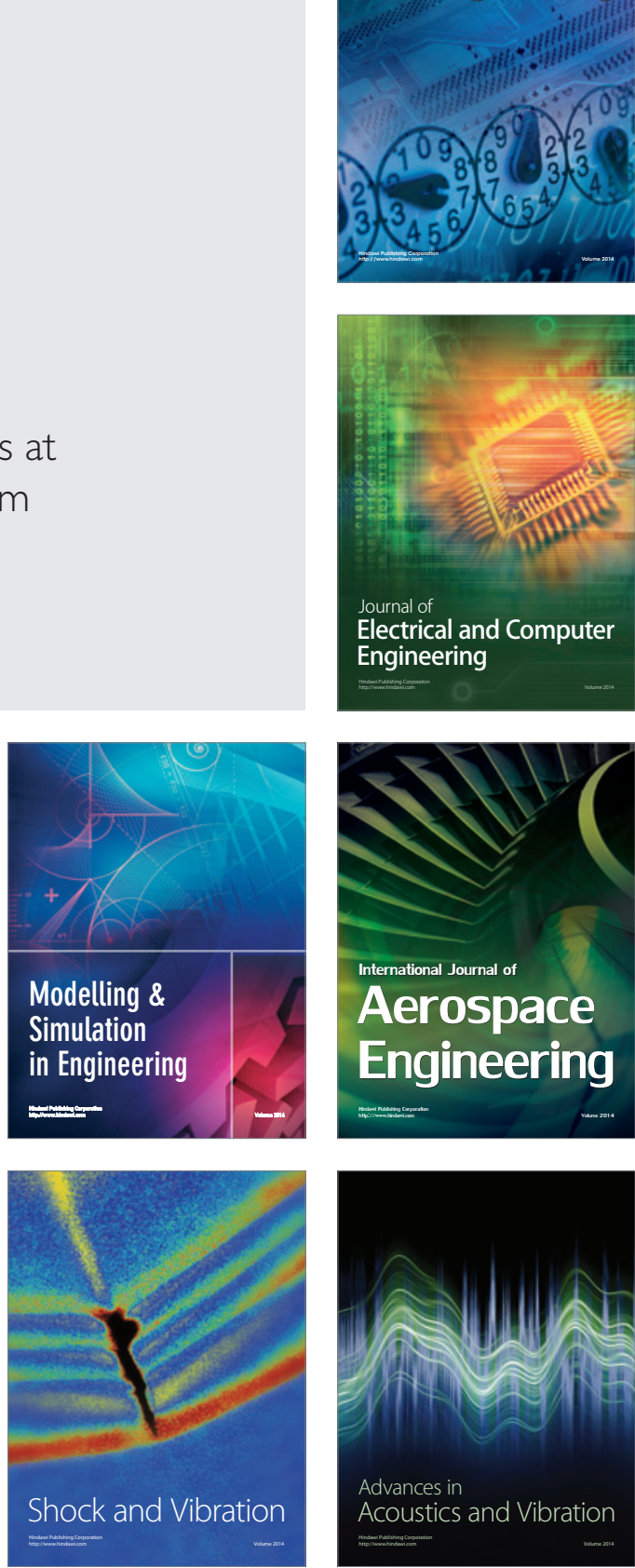